KUCP-0068

hep-th/9407098

July 1994

\title{
Symmetries and tau function of higher dimensional dispersionless integrable hierarchies
}

\author{
Kanehisa Takasaki \\ Department of Fundamental Sciences \\ Faculty of Integrated Human Studies, Kyoto University \\ Yoshida-Nihonmatsu-cho, Sakyo-ku, Kyoto 606, Japan \\ E-mail: takasaki @ jpnyitp.yukawa.kyoto-u.ac.jp
}

\begin{abstract}
A higher dimensional analogue of the dispersionless KP hierarchy is introduced. In addition to the two-dimensional "phase space" variables $(k, x)$ of the dispersionless KP hierarchy, this hierarchy has extra spatial dimensions compactified to a two (or any even) dimensional torus. Integrability of this hierarchy and the existence of an infinite dimensional of "additional symmetries" are ensured by an underlying twistor theoretical structure (or a nonlinear Riemann-Hilbert problem). An analogue of the tau function, whose logarithm gives the $F$ function ("free energy" or "prepotential" in the contest of matrix models and topological conformal field theories), is constructed. The infinite dimensional symmetries can be extended to this tau (or $F$ ) function. The extended symmetries, just like those of the dispersionless KP hierarchy, obey an anomalous commutation relations.
\end{abstract}




\section{Introduction}

Recently we proposed a new family of higher dimensional integrable hierarchies [面]. These hierarchies can be divided into two types. One is formulated in terms of pseudo-differential operators with Moyal algebraic coefficients, and considered to be a higher dimensional analogue of the ordinary KP hierarchy. The other is obtained as classical limit $(\hbar \rightarrow 0)$ of these hierarchies. In this limit, the Moyal algebra turns into a Poisson algebra, and the hierarchies are formulated in terms of Poisson brackets rather than commutators. Because of this, the latter hierarchies are viewed as a higher dimensional analogue of the dispersionless KP hierarchy. The manifold carrying the Moyal or Poisson algebra emerges as "extra spatial dimensions"; this is a reason that we call these hierarchies "higher dimensional".

These higher dimensional integrable hierarchies naturally inherit various characteristics of ordinary integrable hierarchies. In particular, one can develop a Lax formalism and a Riemann-Hilbert problem method in an almost parallel way. The notion of "tau functions" for these hierarchies, however, has remained obscure. Since tau functions are the most fundamental quantities in low dimensional integrable hierarchies [2, 3, 4], extending the theory of tau functions to these new hierarchies will be a crucial issue for better understanding of higher dimensional integrable hierarchies.

As far as we know, no essentially higher dimensional extension of tau functions has been constructed. Those that are already proposed will be presumably interpreted as the tau function of a multi-component KP (or Toda) hierarchy. Another possible candidate of a higher dimensional tau function is the Plebanski function (Kähler potential) $\Omega$ of self-dual Einstein gravity [5]. If, however, one attempts to carefully compare $\Omega$ with, for instance, the tau function $\tau_{d K P}$ of the dispersionless KP hierarchy (and related integrable hierarchies) [6, 7,8$]$, it will turn out that $\Omega$ corresponds to the spatial $\operatorname{logarithmic}$ derivative $\partial_{x} \log \tau_{d K P}$ rather than to $\tau_{d K P}$ itself. This difference is also reflected in their behavior under "additional symmetries" of these systems. Additional symmetries of these systems both carry a Lie algebraic structure related to two-dimensional Poisson algebras, but whereas the Lie algebraic structure of $\Omega$ is the Poisson algebra itself [9, 10, 11, the Lie algebra emerging in $\tau_{d K P}$ is a central extension of the Poisson algebra

[8]. The presence of such a central extension is a common characteristic of tau functions of low dimensional integrable integrable hierarchies. We ex- 
pect that a higher dimensional tau function, if any, should possess a similar property.

As the first step towards such a higher dimensional theory of tau functions, we propose an analogue of $\tau_{d K P}$ for a higher dimensional dispersionless integrable hierarchy. More precisely, it is the $F$ function

$$
F=\log \tau_{d K P}
$$

rather than the tau function $\tau_{d K P}$ itself that plays a fundamental role in the dispersionless KP hierarchy; $F$ is also called the "free energy" or "prepotential" in the context of matrix models and topological conformal field theories. We shall construct a higher dimensional analogue of this $F$ function.

To obtain such a higher dimensional $F$ function, we have to slightly modify our previous construction [1] of higher dimensional integrable hierarchies. Our higher dimensional integrable hierarchies are characterized by a Moyal or Poisson algebra on extra spatial dimensions, which (for simplicity) we assume to be a two-dimensional surface $\Sigma$. In the previous models (planar models), $\Sigma$ is a two-dimensional plane. This is not suited for constructing an $F$ function. We now consider a model (toroidal model) for which $\Sigma$ is a twodimensional torus $T^{2}$. Unlike the planer case, the toroidal Poisson algebra has the linear functional

$$
a \mapsto \int_{T^{2}} \frac{d \theta_{1} d \theta_{2}}{(2 \pi)^{2}} a\left(\theta_{1}, \theta_{2}\right),
$$

where $\left(\theta_{1}, \theta_{2}\right)$ are angle variables on the torus $T^{2}$. This linear functional on the Poisson algebra is, in a sense, a remnant of "trace", because it satisfies the identity

$$
\int_{T^{2}} \frac{d \theta_{1} d \theta_{2}}{(2 \pi)^{2}}\{a, b\}_{\theta}=0
$$

where $\{, \quad\}_{\theta}$ denotes the standard Poisson bracket

$$
\{a, b\}_{\theta}=\frac{\partial a}{\partial \theta_{1}} \frac{\partial b}{\partial \theta_{2}}-\frac{\partial a}{\partial \theta_{2}} \frac{\partial b}{\partial \theta_{1}} .
$$

Actually, the same identity is also satisfied by the Moyal bracket

$$
\{a, b\}_{\hbar, \theta}=\left.\frac{2}{\hbar} \sin \left[\frac{\hbar}{2}\left(\frac{\partial^{2}}{\partial \theta_{1} \partial \theta_{2}^{\prime}}-\frac{\partial^{2}}{\partial \theta_{2} \partial \theta_{1}^{\prime}}\right)\right] a\left(\theta_{1}, \theta_{2}\right) b\left(\theta_{1}^{\prime}, \theta_{2}^{\prime}\right)\right|_{\theta_{1}^{\prime}=\theta_{1}, \theta_{2}^{\prime}=\theta_{2}}
$$


and the above linear functional may be understood as a trace functional on the toroidal Moyal algebra [12]. The above identity for the Poisson bracket is its classical limit. It is this trace functional that allows us to define a higher dimensional $F$ function. In principle, one should be abel to deal with more general models with such a trace functional. The reason that we now focus on the case of $\Sigma=T^{2}$ is rather technical; in that case, the structure of the integrable hierarchy is almost the same as the planar model, so that the construction of the $F$ function relatively simplifies.

The role of such a "trace functional" will become most clear from the point of view of "large- $N$ limit". As stressed in the previous paper [1], our higher dimensional integrable hierarchies may be thought of as "large- $N$ limit" of $N$-component KP hierarchies. The origin of the extra spatial dimensions lies in physicists' observation [13 that the Lie algebra of $N \times N$ matrices turns into a Moyal or Poisson algebra on a two-dimensional surface $\Sigma$ as $N \rightarrow \infty$ :

$$
\operatorname{gl}(N) \underset{N \rightarrow \infty}{\longrightarrow}\left\{\begin{array}{l}
\operatorname{Poisson}(\Sigma) \\
\operatorname{Moyal}(\Sigma)
\end{array}\right.
$$

This observation was a clue to Park's interpretation of self-dual gravity as large- $N$ limit of two-dimensional sigma models [11] and its Moyal algebraic deformations [14]; we applied the same idea to the $N$-component $\mathrm{KP}$ hierarchy. The $N$-component KP hierarchy is formulated in terms of pseudo-differential operators with $\mathrm{gl}(N)$ coefficients. In large- $N$ limit, accordingly, those pseudo-differential operators will be replaced by corresponding quantities (i.e., pseudo-differential operators or phase space functions) with Poisson $(\Sigma)$ or $\operatorname{Moyal}(\Sigma)$ coefficients - this heuristic consideration lies in the heart of our previous work.

Since the notion of tau functions is extended to the $N$-component KP hierarchy [2, 3, 4], it is natural to attempt to consider large- $N$ limit of the tau function of the $N$-component KP hierarchy. Actually, life is not so simple, because the usual definition of a tau function as an infinite determinant [2, [1] or the vacuum expectation value of a free fermion theory [3] is (at least for the moment) not suited for attain this limit.

To overcome this difficulty, we rather resort to an older version of the theory of tau functions developed in the context of "monodromy preserving deformations" [15]. Tau functions are defined therein as a potential of a closed 1-form,

$$
d \log \tau=\alpha=\sum \alpha_{n} d t_{n}
$$


and the coefficients on the right hand side are given by a trace

$$
\alpha_{n}=\operatorname{tr}_{N \times N} A_{n}
$$

of $\mathrm{gl}(N)$-valued functions that are comprised of Laurent coefficients of a particular solution in an associated linear problem. This is also the case for the $N$-component KP hierarchy [3]. In large- $N$ limit, the trace $\operatorname{tr}_{N \times N}$ should turn into the aforementioned trace functional on $\operatorname{Poisson}(\Sigma)$ or $\operatorname{Moyal}(\Sigma)$. More precisely, we have to "renormalize" $\operatorname{tr}_{N \times N}$ by the factor $1 / N$ :

$$
\frac{1}{N} \operatorname{tr}_{N \times N} A \underset{N \rightarrow \infty}{\rightarrow} \operatorname{tr}_{\Sigma} a=\int_{\Sigma} \frac{d \theta_{1} d \theta_{2}}{(2 \pi)^{2}} a\left(\theta_{1}, \theta_{2}\right) .
$$

Combining this relation with the above defining equation of $\log \tau$, one will be naturally led to imagine that a higher dimensional tau function (or $F$ function) is defined in a similar way as above:

$$
d \log \tau=\operatorname{tr}_{\Sigma}(\ldots) \quad\left(\text { or } \quad d F=\operatorname{tr}_{\Sigma}(\ldots)\right) .
$$

We shall indeed define an $F$ function of the toroidal model as such a potential, though the 1-form itself is obtained by guess work (in analogy from the corresponding formula to the dispersionless KP hierarchy) rather than literally chasing this large- $N$ limit (which seems technically difficult).

To confirm that our definition of a higher dimensional $F$ function is a correct one, we construct an infinite dimensional symmetries of the hierarchy, and examine their algebraic properties concerning $F$. The above "trace functional" enters into the construction of symmetries on $F$, too, so that the planar model has to be ruled out again. Apart from this new ingredient, the construction of symmetries is very similar (though considerably complicated) to the case of the dispersionless KP hierarchy. As expected, those symmetries obey anomalous commutation relations and give a central extension of a four-dimensional Poisson algebra.

This paper is organized as follows. Sections 2 and 3 are rather of preliminary nature. In Section 2, we collect basic results from our previous papers [8, 16] and review [17]. These results are presented as a prototype of the contents of subsequent sections. In Section 4, we introduce our new hierarchy and show an underlying twistor theoretical structure ensuring integrability of the hierarchy. Sections 4 and 5 are devoted to presenting our main results. In Section 4, we construct additional symmetries in the language of 
the Lax formalism, and show that their commutation relations obey an underlying Poisson algebraic structure. In Section 5, we define the $F$ function and consider the symmetries of the previous section in the language of this $F$ function. Commutation relations of the extended symmetries are calculated and shown to include an anomalous central term. Section 6 is for concluding remarks and discussions.

\section{Dispersionless KP hierarchy}

\subsection{Extended Lax formalism}

The extended Lax formalism of the dispersionless KP hierarchy consists of the following three equations.

$$
\begin{aligned}
\frac{\partial \mathcal{L}}{\partial t_{n}} & =\left\{\mathcal{B}_{n}, \mathcal{L}\right\}_{k x} \\
\frac{\partial \mathcal{M}}{\partial t_{n}} & =\left\{\mathcal{B}_{n}, \mathcal{M}\right\}_{k x} \\
\{\mathcal{L}, \mathcal{M}\}_{k x} & =1 .
\end{aligned}
$$

The first equation of (2.1) corresponds to the usual Lax representation of the KP hierarchy with an infinite number of time variables $t=\left(t_{1}, t_{2}, \ldots\right)$. The dispersionless analogue $\mathcal{L}$ of the Lax operator $L$ is a Laurent series of the form

$$
\mathcal{L}=k+\sum_{n=1}^{\infty} g_{n+1}(t, x) k^{-n}
$$

and $\{,\}_{k x}$ stands for a Poisson bracket in the two-dimensional "phase space" $(k, x)$,

$$
\{A, B\}_{k x}=\frac{\partial A}{\partial k} \frac{\partial B}{\partial x}-\frac{\partial A}{\partial x} \frac{\partial B}{\partial k} .
$$

The dispersionless analogue $\mathcal{B}_{n}$ of the Zakharov-Shabat operators $B_{n}$ are given by

$$
\mathcal{B}_{n}=\left(\mathcal{L}^{n}\right)_{\geq 0}
$$

and obey the zero-curvature equations

$$
\frac{\partial \mathcal{B}_{m}}{\partial t_{n}}-\frac{\partial \mathcal{B}_{n}}{\partial t_{m}}+\left\{\mathcal{B}_{m}, \mathcal{B}_{n}\right\}_{k x}=0
$$


Here ()$_{\geq 0}$ denotes the projection onto the polynomial part of Laurent series in $k$. Similarly, we shall use ()$_{\leq-1}$ for the complementary projection (i.e., strictly negative power part):

$$
\begin{aligned}
\left(\sum a_{n} k^{n}\right)_{\geq 0} & =\sum_{n \geq 0} a_{n} k^{n}, \\
\left(\sum a_{n} k^{n}\right)_{\leq-1} & =\sum_{n \leq-1} a_{n} k^{n} .
\end{aligned}
$$

The status of the variable $t_{1}$ is special: Since $\mathcal{B}_{1}=k$, the $t_{1}$-dependence of all quantities coincides with the $x$-dependence,

$$
\frac{\partial \mathcal{L}}{\partial t_{1}}=\frac{\partial \mathcal{L}}{\partial x}, \quad \frac{\partial \mathcal{M}}{\partial t_{1}}=\frac{\partial \mathcal{M}}{\partial x},
$$

therefore all quantities of the hierarchy depend on $t_{1}$ and $x$ only through the linear combination $t_{1}+x$. We could have accordingly put $t_{1}=x$, but we rather distinguish between them so as to clarify their roles as time and space variables.

The second equation of (2.1) and the canonical relation (2.2) characterize the dispersionless analogue $\mathcal{M}$ of the Orlov-Shulman operator $M$ [18]:

$$
\mathcal{M}=\sum_{n=1}^{\infty} n t_{n} \mathcal{L}^{n-1}+x+\sum_{n=1}^{\infty} h_{n}(t, x) \mathcal{L}^{-n-1}
$$

Such an extended Lax formalism of the KP hierarchy, as pointed out by Orlov, Shulman and Grinevich [18, 19], provides a very useful framework for describing "additional symmetries" of the KP hierarchy. This is also the case of the dispersionless KP hierarchy. Furthermore, the extended Lax representation of the dispersionless case can also be rewritten into a 2 -form equation of the form

$$
d \mathcal{L} \wedge d \mathcal{M}=d k \wedge d x+\sum_{n=1}^{\infty} d \mathcal{B}_{n} \wedge d t_{n}
$$

where " $d$ " here stands for total differential in $(k, t, x)$. This 2-form equation is a clue for applying Penrose's twistor theoretical approach to the self-dual Einstein equation ("nonlinear graviton construction") [20]. The integrability of the system and the existence of an infinite dimensional symmetries are a consequence of this twistor theoretical structure. 


\subsection{Additional symmetries}

By "symmetries", we mean infinitesimal symmetries. Such symmetries can be most clearly formulated in the abstract language of differential rings. 1 We now introduce two fundamental differential rings.

Let $\mathcal{R}_{0}$ be the differential ring generated by $g_{n}$ and $h_{n}$. As a commutative ring, $\mathcal{R}_{0}$ has generators $g_{n}^{(\alpha)}$ and $h_{n}^{(\alpha)}, \alpha=0,1, \ldots$, which represent $\alpha$-th derivatives of $g_{n}$ and $h_{n}$. We consider them as abstract symbols rather than actual functions. These generators are subject to an infinite set of algebraic equations to be derived from canonical relation (2.2). This ring is further equipped with abstract derivations $\partial$ and $\partial_{n}$ that represent $\partial / \partial x$ and $\partial / \partial t_{n}$. They are to be defined in an obvious way as:

$$
\begin{array}{cl}
\partial g_{n}^{(\alpha)}=g_{n}^{(\alpha+1)}, & \partial h_{n}^{(\alpha)}=h_{n}^{(\alpha+1)}, \\
\partial_{n} g_{n}^{(\alpha)}=\ldots, & \partial_{n} h_{n}^{(\alpha)}=\ldots,
\end{array}
$$

where the right hand side of the last two equations are the same as the right hand side of the equations to be derived from (2.1). Explicit forms are however irrelevant here. The dispersionless KP hierarchy is thus encoded into the abstract structure of the differential ring $\mathcal{R}_{0}$.

Let $\mathcal{R}$ be the extension of this differential ring by variables $t_{n}$ and $x$. For definiteness, we may take the ring of formal power series: $\mathcal{R}=\mathcal{R}_{0}[[t, x]]$. The abstract derivations $\partial$ and $\partial_{n}$ can be uniquely extended onto this larger ring by the obvious rules

$$
\begin{aligned}
\partial x & =1, & \partial t_{m}=0, \\
\partial_{n} x & =0, & \partial_{n} t_{m}=\delta_{n m} .
\end{aligned}
$$

We need such an extended ring in order to define the notion of "additional symmetries". By definition, an additional symmetry (which, from now on, we simply call a symmetry) is a linear map $\delta: \mathcal{R} \rightarrow \mathcal{R}$ that commute with all $\partial$ and $\partial_{n}$ :

$$
[\delta, \partial]=0, \quad\left[\delta, \partial_{n}\right]=0 .
$$

Actually, we only consider a more restricted class of symmetries, i.e., symmetries that leave $x$ and $t_{n}$ invariant:

$$
\delta x=0, \quad \delta t_{n}=0 .
$$

\footnotetext{
${ }^{1} \mathrm{~A}$ differential ring is a $\operatorname{ring} \mathcal{R}$ with a set of abstract derivations $\left\{\partial_{i}\right\}$, i.e., linear maps $\mathcal{R} \rightarrow \mathcal{R}$ obeying the Leibniz rule $\partial_{i}(a b)=\left(\partial_{i} a\right) b+a\left(\partial_{i} b\right), a, b \in \mathcal{R}$.
} 
Such a symmetry represents an "inner" symmetry that commute with spatial and time translations.

This abstract formulation of symmetries can be translated into a more down-to-earth language. Let us consider an infinitesimal transformation $\mathcal{L} \rightarrow$ $\mathcal{L}+\epsilon \delta \mathcal{L}, \mathcal{M} \rightarrow \mathcal{M}+\epsilon \delta \mathcal{M}$ with an infinitesimal parameter $\epsilon$, and look for a condition under which the extended Lax equations are satisfied to the first order of $\epsilon$, i.e.,

$$
\frac{\partial}{\partial t_{n}}(\mathcal{L}+\epsilon \mathcal{L})=\left\{\mathcal{B}_{n}+\epsilon \mathcal{B}_{n}, \mathcal{L}+\epsilon \mathcal{L}\right\}_{k x}+O\left(\epsilon^{2}\right), \quad \text { etc. }
$$

where $\mathcal{B}_{n} \rightarrow \mathcal{B}_{n}+\epsilon \delta \mathcal{B}_{n}$ is understood to be the transformation induced by the relation between $\mathcal{B}_{n}$ and $\mathcal{L}$. Here and in what follows, we again use the explicit notations $\partial / \partial x$ and $\partial / \partial t_{n}$ rather than the abstract ones $\partial$ and $\partial_{n}$. Picking out terms of first order in $\epsilon$, we obtain the following conditions for $\delta \mathcal{L}$ and $\delta \mathcal{M}$ to become a symmetry of the dispersionless KP hierarchy:

$$
\begin{aligned}
\frac{\partial}{\partial t_{n}} \delta \mathcal{L} & =\left\{\delta \mathcal{B}_{n}, \mathcal{L}\right\}_{k x}+\left\{\mathcal{B}_{n}, \delta \mathcal{L}\right\}_{k x} \\
\frac{\partial}{\partial t_{n}} \delta \mathcal{M} & =\left\{\delta \mathcal{B}_{n}, \mathcal{M}\right\}_{k x}+\left\{\mathcal{B}_{n}, \delta \mathcal{M}\right\}_{k x} \\
0 & =\{\delta \mathcal{L}, \mathcal{M}\}_{k x}+\{\mathcal{L}, \delta \mathcal{M}\}_{k x}
\end{aligned}
$$

Recalling that $\delta$ is a derivation on $\mathcal{R}$ (i.e., satisfies the Leibniz rule), these conditions can be written more simply:

$$
\begin{aligned}
\frac{\partial}{\partial t_{n}} \delta \mathcal{L} & =\delta\left(\left\{\mathcal{B}_{n}, \mathcal{L}\right\}_{k x}\right)=\delta \frac{\partial \mathcal{L}}{\partial t_{n}} \\
\frac{\partial}{\partial t_{n}} \delta \mathcal{M} & =\delta\left(\left\{\mathcal{B}_{n}, \mathcal{M}\right\}_{k x}\right)=\delta \frac{\partial \mathcal{M}}{\partial t_{n}} \\
0 & =\delta\{\mathcal{L}, \mathcal{M}\}_{k x} .
\end{aligned}
$$

The first and second equations of the last expression simply mean that $\delta$ and $\partial / \partial_{n}$ commute. Since $\partial / \partial x$ is effectively the same as $\partial / \partial t_{1}, \delta$ also commutes with $\partial / \partial x$. Thus we can reproduce (2.13).

Symmetries satisfying these conditions are explicitly constructed as follows. Given an arbitrary Laurent series

$$
\mathcal{A}=\sum_{i=-\infty}^{\infty} \sum_{j=0}^{\infty} a_{i j} \lambda^{i} \mu^{j},
$$


one can define a derivation $\delta_{\mathcal{A}}$ on $\mathcal{R}$ by the conditions

$$
\begin{aligned}
\delta_{\mathcal{A}} \mathcal{L} & =\left\{\mathcal{A}(\mathcal{L}, \mathcal{M})_{\leq-1}, \mathcal{L}\right\}_{k x} \\
\delta_{\mathcal{A}} \mathcal{M} & =\left\{\mathcal{A}(\mathcal{L}, \mathcal{M})_{\leq-1}, \mathcal{M}\right\}_{k x} \\
\delta_{\mathcal{A}} x & =\delta_{\mathcal{A}} t_{n}=0
\end{aligned}
$$

This additional derivation $\delta_{\mathcal{A}}$ turns out to satisfy the above conditions to satisfy (2.17). Furthermore, these symmetries obey the commutation relations

$$
\left[\delta_{\mathcal{A}}, \delta_{\mathcal{B}}\right]=\delta_{\{\mathcal{A}, \mathcal{B}\}_{\lambda \mu}}
$$

where

$$
\{\mathcal{A}, \mathcal{B}\}_{\lambda \mu}=\frac{\partial \mathcal{A}}{\partial \lambda} \frac{\partial \mathcal{B}}{\partial \mu}-\frac{\partial \mathcal{A}}{\partial \mu} \frac{\partial \mathcal{B}}{\partial \lambda}
$$

This the linear map $\mathcal{A} \mapsto \delta_{\mathcal{A}}$ gives a homomorphism of a two-dimensional Poisson algebra into the algebra of symmetries on $\mathcal{R}$.

As already mentioned, the origin of these symmetries lies in the twistor theoretical structure (and an underlying Riemann-Hilbert problem). This has been discussed in our earlier work on the dispersionless KP (and Toda) hierarchy [8, 21] and its prototype in the self-dual Einstein equation [9, 10, 11]. These symmetries can also be reproduced as a quasi-classical limit of $W_{1+\infty}$ symmetries of the KP hierarchy [16].

\section{3 $\quad$ F function and symmetries}

The $F$ function of the dispersionless KP hierarchy was first introduced for special solutions related to topological conformal field theories [6, 0], then reformulated for general solutions in the following form [B].

In the language of the dispersionless $\mathrm{KP}$ hierarchy, the $\mathrm{F}$ function $F=$ $F(t, x)$ can be characterized as a solution of the equations

$$
\frac{\partial F}{\partial t_{n}}=h_{n}, \quad \frac{\partial F}{\partial x}=h_{1}
$$

or, equivalently, as a (local) potential of a closed 1-form,

$$
d F=\sum_{n=1}^{\infty} h_{n} d t_{n}+h_{1} d x
$$


Frobenius integrability of (2.22), or closedness of the right hand side of (2.23), is indeed ensured by the dispersionless KP hierarchy itself. Now $h_{n}$ 's are derivatives of a single function, $F$. Meanwhile, it is also known that all $g_{n}$ 's are differential polynomials of $h_{n}$ 's; $g_{n}$ 's, too, can be reproduced from $F$. Thus $F$ plays the role of a "generating function". This role of $F$ is parallel to the tau function of the full KP hierarchy.

The $F$ function has another characterization. The full KP hierarchy can be reformulated so as to include a Planck constant $\hbar$. The tau function, too, then depends on $\hbar$ as $\tau=\tau(\hbar, t, x)$. In the limit of $\hbar \rightarrow 0, \tau$ behaves as

$$
\tau(\hbar, t, x)=\exp \left(-\hbar^{-2} F(t, x)+O\left(\hbar^{-1}\right)\right),
$$

thus $F$ emerges as the leading term of $\hbar$-expansion of $\log \tau$.

In the context of differential rings, defining $F$ as above amounts to extending the differential rings $\mathcal{R}_{0}$ and $\mathcal{R}$ into the rings $\mathcal{R}_{0}[F]$ and $\mathcal{R}[F]$ of polynomials in $F$ (with coefficients taken from $\mathcal{R}_{0}$ and $\mathcal{R}$ ). One should then reinterpret Eqs. (2.22) and (2.23) as defining an extension of the abstract derivations $\partial(\leftrightarrow \partial / \partial x)$ and $\partial_{n}\left(\leftrightarrow \partial / \partial t_{n}\right)$ of $\mathcal{R}$ onto these larger rings; Frobenius integrability condition (or closedness of the 1-form) is nothing more than a consistency condition for this extension of derivations.

This point of view also applies to symmetries. Indeed the symmetries $\delta_{\mathcal{A}}$ on $\mathcal{R}$, too, turn out to have a consistent extension onto $\mathcal{R}$ as follows:

$$
\delta_{A} F=-\operatorname{res}_{\lambda} \int_{0}^{\mathcal{M}(\lambda)} d \mu \mathcal{A}(\lambda, \mu)
$$

where " $\operatorname{res}_{\lambda}$ " means the formal residue with respect to $\lambda$ :

$$
\underset{\lambda}{\operatorname{res}} \sum a_{n} \lambda^{n}=a_{-1}
$$

Note that the above formula can also be written as a double integral over a domain of a two-dimensional "phase space" (a cylinder) with coordinates $(\lambda, \mu)$ :

$$
\delta_{A} F=-\oint \frac{d \lambda}{2 \pi i} \int_{0}^{\mathcal{M}(\lambda)} d \mu \mathcal{A}(\lambda, \mu)
$$

where the path of the $\lambda$-integral is understood to be a small loop around $\lambda=\infty$. It is interesting that this resembles a "fermi fluid" picture of $c=1$ matrix models [22]. We shall discuss this issue in more detail in the final section. 
Consistency conditions of the above extension of $\delta_{\mathcal{A}}$ are given by the equations

$$
\begin{aligned}
\frac{\partial}{\partial t_{n}} \delta_{\mathcal{A}} F & =\delta_{\mathcal{A}} \frac{\partial F}{\partial t_{n}} \quad\left(=\delta_{\mathcal{A}} h_{n}\right) \\
\frac{\partial}{\partial x} \delta_{\mathcal{A}} F & =\delta_{\mathcal{A}} \frac{\partial F}{\partial x} \quad\left(=\delta_{\mathcal{A}} h_{1}\right),
\end{aligned}
$$

which simply say that $\delta_{\mathcal{A}}$ commutes with $\partial / \partial t_{n}$ and $\partial / \partial x$ as derivations on $\mathcal{R}[F]$. One can indeed prove that (2.25) satisfies these conditions.

The extended symmetries obey the "anomalous" commutation relations

$$
\left[\delta_{\mathcal{A}}, \delta_{\mathcal{B}}\right]=\delta_{\{\mathcal{A}, \mathcal{B}\}_{\lambda \mu}}+c(\mathcal{A}, \mathcal{B}) \partial_{F}
$$

where $c(\mathcal{A}, \mathcal{B})$ is a 2-cocycle of the two-dimensional Poisson algebra,

$$
c(\mathcal{A}, \mathcal{B})=\underset{\lambda}{\operatorname{res}} A(\lambda, 0) \frac{\partial \mathcal{B}(\lambda, 0)}{\partial \lambda}
$$

and $\partial_{F}$ is yet another abstract derivation on $\mathcal{R}$ defined by

$$
\partial_{F}(F)=1, \quad \partial_{F}\left(g_{n}^{(\alpha)}\right)=\partial_{F}\left(h_{n}^{(\alpha)}\right)=\partial_{F}\left(t_{n}\right)=\partial_{F}(x)=0 .
$$

Actually, (2.29) is nothing but (2.20) plus the following relation:

$$
\left[\delta_{\mathcal{A}}, \delta_{\mathcal{B}}\right] F=\delta_{\{\mathcal{A}, \mathcal{B}\}_{\lambda \mu}} F+c(\mathcal{A}, \mathcal{B}) \text {. }
$$

Note that $\partial_{F}$ commutes with all other derivations $\partial / \partial t_{n}, \partial / \partial x$ and $\delta_{\mathcal{A}}$. Thus the extended symmetries obey a central extension of the two-dimensional Poisson algebra.

\section{Toroidal model of higher dimensional dis- persionless integrable hierarchies}

\subsection{Formulation of toroidal model}

We now introduce the toroidal model of higher dimensional dispersionless integrable hierarchies. The construction is very similar to the planar model in our previous work [1]. 
Let $\theta=\left(\theta_{1}, \theta_{2}\right)$ be angle variables of a two-dimensional torus $T^{2}\left(\theta_{j} \sim\right.$ $\theta_{j}+2 \pi n, n=$ integer, $\left.j=1,2\right)$. Any function $A=A(\theta)$ on $T^{2}$ can be expanded into a Fourier series $(i=\sqrt{-1})$,

$$
A=\sum_{\alpha_{1}, \alpha_{2}=-\infty}^{\infty} a_{\alpha_{1} \alpha_{2}} e^{i\left(\alpha_{1} \theta_{1}+\alpha_{2} \theta_{2}\right)} .
$$

For such functions, we define the Poisson bracket

$$
\{A, B\}_{\theta}=\frac{\partial A}{\partial \theta_{1}} \frac{\partial B}{\partial \theta_{2}}-\frac{\partial A}{\partial \theta_{2}} \frac{\partial B}{\partial \theta_{1}} .
$$

Furthermore, if $A$ and $B$ also depend on two other variables $(k, x)$ (which are "phase space variables" of the dispersionless KP hierarchy), we define the four-dimensional Poisson bracket

$$
\{A, B\}=\{A, B\}_{k x}+\{A, B\}_{\theta} .
$$

The toroidal model is defined by the Lax equations

$$
\begin{array}{rlrl}
\frac{\partial \mathcal{L}}{\partial t_{n \alpha}} & =\left\{\mathcal{B}_{n \alpha}, \mathcal{L}\right\}, & & \frac{\partial \mathcal{M}}{\partial t_{n \alpha}}=\left\{\mathcal{B}_{n \alpha}, \mathcal{M}\right\}, \\
\frac{\partial \mathcal{U}}{\partial t_{n \alpha}}=\left\{\mathcal{B}_{n \alpha}, \mathcal{U}\right\}, & \frac{\partial \mathcal{V}}{\partial t_{n \alpha}}=\left\{\mathcal{B}_{n \alpha}, \mathcal{V}\right\},
\end{array}
$$

and the canonical relations

$$
\begin{aligned}
\{\mathcal{L}, \mathcal{M}\} & =\{\mathcal{U}, \mathcal{V}\}=1 \\
\{\mathcal{L}, \mathcal{U}\} & =\{\mathcal{L}, \mathcal{V}\}=\{\mathcal{M}, \mathcal{U}\}=\{\mathcal{M}, \mathcal{V}\}=0
\end{aligned}
$$

The time variables $t=\left(t_{n \alpha}\right)$ have a double index $(n, \alpha)$ with $n=0,1, \ldots$ and $\alpha=0, \pm 1, \pm 2, \ldots$ In addition to the analogues $\mathcal{L}$ and $\mathcal{M}$ of the LaxOrlov-Shulman functions in the dispersionless KP hierarchy, we now have two extra functions $\mathcal{U}$ and $\mathcal{V}$ that correspond to the two extra spatial dimensions. These four functions are assumed to be Laurent-Fourier series of the form

$$
\begin{aligned}
\mathcal{L} & =k+\sum_{n=1}^{\infty} g_{n+1}(t, x, \theta) k^{-n} \\
\mathcal{M} & =\sum_{n, \alpha} n t_{n \alpha} \mathcal{L}^{n-1} e^{i \alpha \mathcal{U}}+x+\sum_{n=1}^{\infty} h_{n}(t, x, \theta) \mathcal{L}^{-n-1}
\end{aligned}
$$




$$
\begin{aligned}
& \mathcal{U}=\theta_{1}+\sum_{n=1}^{\infty} u_{n}(t, x, \theta) \mathcal{L}^{-n} \\
& \mathcal{V}=\sum_{n, \alpha} i \alpha t_{n \alpha} \mathcal{L}^{n} e^{i \alpha \mathcal{U}}+\theta_{2}+\sum_{n=1}^{\infty} v_{n}(t, x, \theta) \mathcal{L}^{-n}
\end{aligned}
$$

where the coefficients $g_{n}, h_{n}, u_{n}$ and $v_{n}$ are required to be functions on $T^{2}$ (i.e., doubly periodic). Note that $\mathcal{U}$ and $\mathcal{V}$ themselves are not doubly periodic (because of the presence of the $\theta_{1}$ and $\theta_{2}$ terms on the right hand side), but their exponentials $e^{i \alpha \mathcal{U}}$ are doubly periodic. Finally, $\mathcal{B}_{n \alpha}$ are given by

$$
\mathcal{B}_{n \alpha}=\left(\mathcal{L}^{n} e^{i \alpha \mathcal{U}}\right)_{\geq 0}
$$

where ()$_{\geq 0}$ and ()$_{\leq-1}$ are the same as those of the dispersionless KP hierarchy, i.e., the projections onto nonnegative and negative powers of $k$.

A few technical remarks are now in order:

1. The construction of the toroidal model is almost parallel to the planar model. A main difference is that whereas the planar model is based on Tayler series, the toroidal model uses Fourier series. In the planar model, the angle variables $\left(\theta_{1}, \theta_{2}\right)$ are replaced by planar coordinates $(y, z)$, and the counterparts of $\mathcal{L}, \mathcal{M}, \mathcal{U}$ and $\mathcal{V}$ are assumed to have the following expansion:

$$
\begin{aligned}
\mathcal{L} & =k+\sum_{n=1}^{\infty} g_{n+1}(t, x, y, z) k^{-n} \\
\mathcal{M} & =\sum_{n, \alpha} n t_{n \alpha} \mathcal{L}^{n-1} \mathcal{U}^{\alpha}+x+\sum_{n=1}^{\infty} h_{n}(t, x, y, z) \mathcal{L}^{-n-1} \\
\mathcal{U} & =y+\sum_{n=1}^{\infty} u_{n}(t, x, y, z) \mathcal{L}^{-n} \\
\mathcal{V} & =\sum_{n, \alpha} \alpha t_{n \alpha} \mathcal{L}^{n} \mathcal{U}^{\alpha-1}+z+\sum_{n=1}^{\infty} v_{n}(t, x, y, z) \mathcal{L}^{-n}
\end{aligned}
$$

Furthermore, the second index $\alpha$ is restricted to nonnegative values $\alpha=$ $0,1, \ldots$, and $\mathcal{B}_{n \alpha}$ are given by

$$
\mathcal{B}_{n \alpha}=\left(\mathcal{L}^{n} \mathcal{U}^{\alpha}\right)_{\geq 0}
$$

Apart from this difference, both hierarchies can be treated in much the same way. 
2. It is easy to see that the above extended Lax system indeed gives a well defined set of flows in the space of four functions $(\mathcal{L}, \mathcal{M}, \mathcal{U}, \mathcal{V})$. First, by the definition of $\mathcal{B}_{n \alpha}$ and the canonical relations among $(\mathcal{L}, \mathcal{M}, \mathcal{U}, \mathcal{V})$, the Lax equations of $\mathcal{L}$ and $\mathcal{U}$ can be rewritten

$$
\begin{aligned}
\frac{\partial \mathcal{L}}{\partial t_{n \alpha}} & =-\left\{\mathcal{B}_{n \alpha}^{-}, \mathcal{L}\right\} \\
\frac{\partial \mathcal{U}}{\partial t_{n \alpha}} & =-\left\{\mathcal{B}_{n \alpha}^{-}, \mathcal{U}\right\}
\end{aligned}
$$

where we have defined

$$
\mathcal{B}_{n \alpha}^{-}=\left(\mathcal{L}^{n} e^{i \alpha \mathcal{U}}\right)_{\leq-1} .
$$

Both hand sides of these equations contain only negative powers of $k$, and one can read off a closed set of equations of motions for $g_{n}$ and $u_{n}$ therefrom. Second, as for $\mathcal{M}$ and $\mathcal{V}$, let us drop the first part containing $t$ 's and consider

$$
\begin{aligned}
\frac{\partial \mathcal{M}^{\prime}}{\partial t_{n \alpha}} & =\left\{\mathcal{B}_{n \alpha}, \mathcal{M}^{\prime}\right\}-b \mathcal{L}^{n-1} e^{i \alpha \mathcal{U}}=\left\{\mathcal{B}_{n \alpha}^{-}, \mathcal{M}^{\prime}\right\}, \\
\frac{\partial \mathcal{V}^{\prime}}{\partial t_{n \alpha}} & =\left\{\mathcal{B}_{n \alpha}, \mathcal{V}^{\prime}\right\}-i \alpha \mathcal{L}^{n} e^{i \alpha \mathcal{U}}=\left\{\mathcal{B}_{n \alpha}^{-}, \mathcal{V}^{\prime}\right\}
\end{aligned}
$$

These equations, too, contain only negative powers of $k$, and give a set of equations of motions for $h_{n}$ and $v_{n}$. Third, in terms of these modified quantities, canonical relations (3.5) become

$$
\begin{aligned}
\left\{\mathcal{L}, \mathcal{M}^{\prime}\right\} & =\left\{\mathcal{U}, \mathcal{V}^{\prime}\right\}=1 \\
\{\mathcal{L}, \mathcal{U}\} & =\left\{\mathcal{L}, \mathcal{V}^{\prime}\right\}=\left\{\mathcal{M}^{\prime}, \mathcal{U}\right\}=\left\{\mathcal{M}^{\prime}, \mathcal{V}^{\prime}\right\}=0
\end{aligned}
$$

These relations have to be imposed on the above equations of motion for $\left(\mathcal{L}, \mathcal{M}^{\prime}, \mathcal{U}, \mathcal{V}^{\prime}\right)$. Since the above equations of motion take the form of "Liouville flows" of classical mechanics, the last constraints are indeed consistent with the flows (i.e., preserved for all time if satisfied at the initial time $t=0$ ).

3 . One can derive the zero-curvature equations

$$
\frac{\partial \mathcal{B}_{m \alpha}}{\partial t_{n \beta}}-\frac{\partial \mathcal{B}_{n \beta}}{\partial t_{m \alpha}}+\left\{\mathcal{B}_{m \alpha}, \mathcal{B}_{n \alpha}\right\}=0
$$

from the Lax equations of $\mathcal{L}$ and $\mathcal{U}$. This can be seen by the same method as used in the full and dispersionless KP hierarchy as follows: Note that any 
monomial of $\mathcal{L}$ and $e^{i \mathcal{U}}$ obeys the same Lax equations:

$$
\frac{\partial}{\partial t_{n \beta}}\left(\mathcal{L}^{m} e^{i \alpha \mathcal{U}}\right)=\left\{\mathcal{B}_{n \beta}, \mathcal{L}^{m} e^{i \alpha \mathcal{U}}\right\}
$$

Subtracting from this the same equation with $(m, \alpha)$ and $(n, \beta)$ interchanged, one has

$$
\frac{\partial}{\partial t_{n \beta}}\left(\mathcal{L}^{m} e^{i \alpha \mathcal{U}}\right)-\frac{\partial}{\partial t_{m \alpha}}\left(\mathcal{L}^{n} e^{i \beta \mathcal{U}}\right)=\left\{\mathcal{B}_{n \beta}, \mathcal{L}^{m} e^{i \alpha \mathcal{U}}\right\}-\left\{\mathcal{B}_{m \alpha}, \mathcal{L}^{n} e^{i \beta \mathcal{U}}\right\}
$$

Let us consider both hand sides in more detail. Since $\mathcal{L}^{m} e^{i \alpha \mathcal{U}}=\mathcal{B}_{m \alpha}+\mathcal{B}_{m \alpha}^{-}$, LHS becomes a sum of two parts that consist of only nonnegative or negative powers of $k$ :

$$
\text { LHS of }(3.16)=\left(\frac{\partial \mathcal{B}_{m \alpha}}{\partial t_{n \beta}}-\frac{\partial \mathcal{B}_{n \beta}}{\partial t_{m \alpha}}\right)+\left(\frac{\partial \mathcal{B}_{m \alpha}^{-}}{\partial t_{n \beta}}-\frac{\partial \mathcal{B}_{n \beta}^{-}}{\partial t_{m \alpha}}\right) .
$$

Similarly, recalling the Poisson commutativity of $\mathcal{L}$ and $\mathcal{U}$ again, one can rewrite the right hand side as:

$$
\begin{aligned}
\operatorname{RHS} \text { of }(3.16)= & \left\{\mathcal{B}_{n \beta}, \mathcal{L}^{m} e^{i \alpha \mathcal{U}}\right\}+\left\{\mathcal{B}_{m \alpha}^{-}, \mathcal{L}^{n} e^{i \beta \mathcal{U}}\right\} \\
= & \left\{\mathcal{B}_{n \beta}, \mathcal{B}_{m \alpha}\right\}+\left\{\mathcal{B}_{n \beta}, \mathcal{B}_{m \alpha}^{-}\right\}+\left\{\mathcal{B}_{m \alpha}^{-}, \mathcal{B}_{n \beta}\right\}+\left\{\mathcal{B}_{m \alpha}^{-}, \mathcal{B}_{n \beta}^{-}\right\} \\
= & \left\{\mathcal{B}_{n \beta}, \mathcal{B}_{m \alpha}\right\}+\left\{\mathcal{B}_{m \alpha}^{-}, \mathcal{B}_{n \beta}^{-}\right\} .
\end{aligned}
$$

Thus both hand sides become a sum of two parts each of which contains only nonnegative or negative powers of $k$. The ()$_{\geq 0}$ part gives zero-curvature equation (3.14), whereas the ()$_{\leq-1}$ part yields its "dual" form,

$$
\frac{\partial \mathcal{B}_{m \alpha}^{-}}{\partial t_{n \beta}}-\frac{\partial \mathcal{B}_{n \beta}^{-}}{\partial t_{m \alpha}}-\left\{\mathcal{B}_{m \alpha}^{-}, \mathcal{B}_{n \alpha}^{-}\right\}=0
$$

\subsection{Integrability, twistor theory and Riemann-Hilbert problem}

Just as in the case of the planar model, integrability of this new hierarchy can be explained in two different ways.

One way is to construct a new hierarchy in which the Poisson bracket is replaced by a Moyal bracket, and to resort to integrability of the latter. 
The original model can be reproduced in the classical limit $\hbar \rightarrow 0$. In our previous paper [1], the planar model was treated exactly along this line of approach.

Another way is to search for a twistor theoretical representation (or an equivalent Riemann-Hilbert problem) of the system. This method relies on the existence of a 2-form equation like Eq. (2.10) of the dispersionless KP hierarchy. One can indeed find such a 2-form equation as follows.

Proposition 1 The extended Lax system of the toroidal model is equivalent to the 2-form equation

$$
d \mathcal{L} \wedge d \mathcal{M}+d \mathcal{U} \wedge d \mathcal{V}=d k \wedge d x+d \theta_{1} \wedge d \theta_{2}+\sum_{n, \alpha} d \mathcal{B}_{n \alpha} \wedge d t_{n \alpha}
$$

A proof is given in the next subsection. We here briefly show why this leads to integrability of the model. Basic ieas are the same as in the case of the self-dual Einstein equation [9, 10] and subsequent applications to the dispersionless KP and Toda hierarchies [8, 21], the toroidal model of higher dimensional dispersionless hierarchy [1], etc.

Let $\omega$ denote the right hand side of (3.20). Obviously $\omega$ is a closed form on the $(t, k, x, \theta)$ space,

$$
d \omega=0 .
$$

Furthermore, although the third order exterior power does not vanish, $\omega \wedge$ $\omega \wedge \omega \neq 0$, the fourth one vanishes:

$$
\omega \wedge \omega \wedge \omega \wedge \omega=0
$$

(One can prove this using zero-curvature equations (3.14).) Therefore, by a theorem of Darboux in symplectic geometry, $\omega$ is a degenerate symplectic form and has a local expression

$$
\omega=d \mathcal{P}_{1} \wedge d \mathcal{Q}_{1}+d \mathcal{P}_{2} \wedge d \mathcal{Q}_{2}
$$

with suitable four functions $\left(\mathcal{P}_{1}, \mathcal{Q}_{1}, \mathcal{P}_{2}, \mathcal{Q}_{2}\right)$ (Darboux variables). The above proposition shows that $(\mathcal{L}, \mathcal{M}, \mathcal{U}, \mathcal{V})$ give such a Darboux variable system, which is valid in a neighborhood of $k=\infty$ (i.e., gives a set of complex analytic functions defined in a neighborhood of $k=\infty$ except at $k=\infty$ itself). In fact, the theorem of Darboux ensures only local existence, and 
one can find another Darboux variable system, say $(\overline{\mathcal{L}}, \overline{\mathcal{M}}, \overline{\mathcal{U}}, \overline{\mathcal{V}})$, that are similarly valid in a neighborhood of $k=0$.

Suppose now that the domains $D$ and $\bar{D}$ of these two Darboux variable systems in the $k$ plane (or Riemann sphere) are large enough to cover the whole Riemann sphere. (This is indeed a generic situation of Penrose's nonlinear graviton construction.) The two Darboux variable systems are then linked, via $\omega$, as

$$
d \mathcal{L} \wedge d \mathcal{M}+d \mathcal{U} \wedge d \mathcal{V}=\omega=d \overline{\mathcal{L}} \wedge d \overline{\mathcal{M}}+d \overline{\mathcal{U}} \wedge d \overline{\mathcal{V}} .
$$

This implies that they are functionally related,

$$
\begin{aligned}
\overline{\mathcal{L}}=f_{1}(\mathcal{L}, \mathcal{M}, \mathcal{U}, \mathcal{V}), & \overline{\mathcal{M}}=f_{2}(\mathcal{L}, \mathcal{M}, \mathcal{U}, \mathcal{V}), \\
\overline{\mathcal{U}}=f_{3}(\mathcal{L}, \mathcal{M}, \mathcal{U}, \mathcal{V}), & \overline{\mathcal{V}}=f_{4}(\mathcal{L}, \mathcal{M}, \mathcal{U}, \mathcal{V}),
\end{aligned}
$$

and that the quartet $f=\left(f_{1}, f_{2}, f_{3}, f_{4}\right)$ becomes a four-dimensional symplectic diffeomorphism $(\lambda, \mu, u, v) \mapsto f(\lambda, \mu, u, v)$, i.e., satisfies the symplectic condition

$$
d f_{1} \wedge d f_{2}+d f_{3} \wedge d f_{4}=d \lambda \wedge d \mu+d u \wedge d v .
$$

In the geometric language of twistor theory [20], this symplectic diffeomorphism may be interpreted as a transition function between two local coordinate patches of a four-dimensional complex manifold (twistor space); Eq. (3.25) then define a family of holomorphic curves in the twistor space. From the point of view of the theory of integrable systems, Eq. (3.25) may be thought of as a kind of nonlinear Riemann-Hilbert problem.

This Riemann-Hilbert problem, like those for the other equations mentioned above, is ensured to have a unique solution as far as $f$ is sufficiently close to the identity diffeomorphism. (Actually, unique solvability is also preserved under small perturbations from any soluble data. In the language of twistor theory, this is a consequence of the Kodaira-Spencer theory [20].) This means that there is a one-to-one correspondence between a solution and its twistor data. Such a correspondence lies in the heart of integrable nature of almost all integrable systems. In this sense, the new hierarchy, too, may be called "integrable". 


\subsection{Proof of Proposition}

We give a proof of Proposition 11. The proof is somewhat lengthy and technical, but basic ideas are mostly similar to the case of the dispersionless KP hierarchy.

Let us first derive the extended Lax system from the 2-form equation. Note that the total differentials $d \mathcal{L}$ etc. can be expanded as

$$
d \mathcal{L}=\frac{\partial \mathcal{L}}{\partial k} d k+\frac{\partial \mathcal{L}}{\partial x} d x+\frac{\partial \mathcal{L}}{\partial \theta_{1}} d \theta_{1}+\frac{\partial \mathcal{L}}{\partial \theta_{1}} d \theta_{2}+\sum_{n, \alpha} \frac{\partial \mathcal{L}}{\partial t_{n \alpha}} d t_{n \alpha}, \ldots
$$

Substituting this expression, one can write the the left hand side of the 2form equation as a linear combination of the basic 2 -forms $d k \wedge d x$, etc. We now pick out coefficients for each basic 2-form.

First, modulo terms including $d t$ 's, the 2 -form equation can be written

$$
\left.d \mathcal{L} \wedge d \mathcal{M}+d \mathcal{U} \wedge d \mathcal{V}=d k \wedge d x+d \theta_{1} \wedge d \theta_{2}+\text { (terms including } d t^{\prime} \text { s }\right) .
$$

This shows that the four-dimensional map $\left(k, x, \theta_{1}, \theta_{2}\right) \mapsto(\mathcal{L}, \mathcal{M}, \mathcal{U}, \mathcal{V})$ is a symplectic diffeomorphism with parameters $t_{n \alpha}$. Therefore canonical relations (3.5) follow.

Second, from terms of the form $d k \wedge d t_{n \alpha}, d x \wedge d t_{n \alpha}$ and $d \theta_{j} \wedge d t_{n \alpha}$, respectively, the following equations emerge:

$$
\begin{aligned}
\frac{\partial(\mathcal{L}, \mathcal{M})}{\partial\left(k, t_{n \alpha}\right)}+\frac{\partial(\mathcal{U}, \mathcal{V})}{\partial\left(k, t_{n \alpha}\right)} & =\frac{\partial \mathcal{B}_{n \alpha}}{\partial k} \\
\frac{\partial(\mathcal{L}, \mathcal{M})}{\partial\left(x, t_{n \alpha}\right)}+\frac{\partial(\mathcal{U}, \mathcal{V})}{\partial\left(x, t_{n \alpha}\right)} & =\frac{\partial \mathcal{B}_{n \alpha}}{\partial x} \\
\frac{\partial(\mathcal{L}, \mathcal{M})}{\partial\left(\theta_{j}, t_{n \alpha}\right)}+\frac{\partial(\mathcal{U}, \mathcal{V})}{\partial\left(\theta_{j}, t_{n \alpha}\right)} & =\frac{\partial \mathcal{B}_{n \alpha}}{\partial \theta_{j}}
\end{aligned}
$$

where we have used the notation

$$
\frac{\partial(A, B)}{\partial(p, q)}=\frac{\partial A}{\partial p} \frac{\partial B}{\partial q}-\frac{\partial A}{\partial p} \frac{\partial B}{\partial q} .
$$

These four equations give a linear system of equations for the $t$-derivatives of $\mathcal{L}, \mathcal{M}, \mathcal{U}$ and $\mathcal{V}$ :

$$
\left(\frac{\partial \mathcal{M}}{\partial t_{n \alpha}},-\frac{\partial \mathcal{L}}{\partial t_{n \alpha}}, \frac{\partial \mathcal{V}}{\partial t_{n \alpha}},-\frac{\partial \mathcal{U}}{\partial t_{n \alpha}}\right) J=\left(\frac{\partial \mathcal{B}_{n \alpha}}{\partial k}, \frac{\partial \mathcal{B}_{n \alpha}}{\partial x}, \frac{\partial \mathcal{B}_{n \alpha}}{\partial \theta_{1}}, \frac{\partial \mathcal{B}_{n \alpha}}{\partial \theta_{2}}\right)
$$


where the matrix $J$ is given by

$$
J=\left(\begin{array}{cccc}
\frac{\partial \mathcal{L}}{\partial k} & \frac{\partial \mathcal{L}}{\partial x} & \frac{\partial \mathcal{L}}{\partial \theta_{1}} & \frac{\partial \mathcal{L}}{\partial \theta_{2}} \\
\frac{\partial \mathcal{M}}{\partial k} & \frac{\partial \mathcal{M}}{\partial x} & \frac{\partial \mathcal{M}}{\partial \theta_{1}} & \frac{\partial \mathcal{M}}{\partial \theta_{2}} \\
\frac{\partial \mathcal{U}}{\partial k} & \frac{\partial \mathcal{U}}{\partial x} & \frac{\partial \mathcal{U}}{\partial \theta_{1}} & \frac{\partial \mathcal{U}}{\partial \theta_{2}} \\
\frac{\partial \mathcal{V}}{\partial k} & \frac{\partial \mathcal{V}}{\partial x} & \frac{\partial \mathcal{V}}{\partial \theta_{1}} & \frac{\partial \mathcal{V}}{\partial \theta_{2}}
\end{array}\right)
$$

Since the four-dimensional map $\left(k, x, \theta_{1}, \theta_{2}\right) \mapsto(\mathcal{L}, \mathcal{M}, \mathcal{U}, \mathcal{V})$ is symplectic, this matrix $J$ is a symplectic matrix, and the inverse matrix can be written

$$
J^{-1}=\left(\begin{array}{rrrr}
\frac{\partial \mathcal{M}}{\partial x} & -\frac{\partial \mathcal{L}}{\partial x} & \frac{\partial \mathcal{V}}{\partial x} & -\frac{\partial \mathcal{U}}{\partial x} \\
-\frac{\partial \mathcal{M}}{\partial k} & \frac{\partial \mathcal{L}}{\partial k} & -\frac{\partial \mathcal{V}}{\partial k} & \frac{\partial \mathcal{U}}{\partial k} \\
\frac{\partial \mathcal{M}}{\partial \theta_{2}} & -\frac{\partial \mathcal{L}}{\partial \theta_{2}} & \frac{\partial \mathcal{V}}{\partial \theta_{2}} & -\frac{\partial \mathcal{U}}{\partial \theta_{2}} \\
-\frac{\partial \mathcal{M}}{\partial \theta_{1}} & \frac{\partial \mathcal{L}}{\partial \theta_{1}} & -\frac{\partial \mathcal{V}}{\partial \theta_{1}} & \frac{\partial \mathcal{U}}{\partial \theta_{1}}
\end{array}\right)
$$

Therefore the above equations can be solved for $\mathcal{L}, \mathcal{M}, \mathcal{U}$ and $\mathcal{V}$ as follows:

$$
\left(\frac{\partial \mathcal{M}}{\partial t_{n \alpha}},-\frac{\partial \mathcal{L}}{\partial t_{n \alpha}}, \frac{\partial \mathcal{V}}{\partial t_{n \alpha}},-\frac{\partial \mathcal{U}}{\partial t_{n \alpha}}\right)=\left(\frac{\partial \mathcal{B}_{n \alpha}}{\partial k}, \frac{\partial \mathcal{B}_{n \alpha}}{\partial x}, \frac{\partial \mathcal{B}_{n \alpha}}{\partial \theta_{1}}, \frac{\partial \mathcal{B}_{n \alpha}}{\partial \theta_{2}}\right) J^{-1}
$$

Plugging the previous expression of $J^{-1}$ and calculating the right hand side explicitly, one will find that this gives Lax equations (3.4) exactly.

Finally, terms of the form $d t_{n \beta} \wedge d t_{m \alpha}$ give

$$
\frac{\partial(\mathcal{L}, \mathcal{M})}{\partial\left(t_{m \alpha}, t_{n \beta}\right)}+\frac{\partial(\mathcal{U}, \mathcal{V})}{\partial\left(t_{m \alpha}, t_{n \beta}\right)}=\frac{\partial \mathcal{B}_{n \beta}}{\partial t_{m \alpha}}-\frac{\partial \mathcal{B}_{m \alpha}}{\partial t_{n \beta}}
$$

Using (3.4,3.5), which we have proven, one can easily see that the left hand side coincides with $\left\{\mathcal{B}_{m \alpha}, \mathcal{B}_{n \beta}\right\}$. The above equation therefore turns out to be zero-curvature equation (3.14). Thus all equations of the extended Lax system can be derived from the 2-form equation.

One can easily see that the converse is also true. Recall that zerocurvature equations (3.14) can be derived from the Lax equations. This ensures the vanishing of $d t_{n \beta} \wedge d t_{m \beta}$-part in the 2 -form equation. Having this, one can actually check that the above derivation is reversible. 


\subsection{Another expression of hierarchy}

Both the planar and toroidal models are formulated in terms of the four fundamental quantities $(\mathcal{L}, \mathcal{M}, \mathcal{U}, \mathcal{V})$. This expression is particularly suited for understanding the twistor theoretical meaning and the underlying RiemannHilbert problem. In the subsequent sections, however, another expression of the same hierarchy becomes more useful.

Let us introduce a new variable $\lambda$ ("spectral parameter") and define the following Laurent series of $\lambda$ :

$$
\begin{aligned}
\mathcal{M}(\lambda) & =\sum_{n, \alpha} n t_{n \alpha} \lambda^{n-1} e^{i \alpha \mathcal{U}(\lambda)}+x+\sum_{n=1}^{\infty} h_{n} \lambda^{-n-1}, \\
\mathcal{U}(\lambda) & =\theta_{1}+\sum_{n=1}^{\infty} u_{n} \lambda^{-n} \\
\mathcal{V}(\lambda) & =\sum_{n, \alpha} i \alpha t_{n \alpha} \lambda^{n} e^{i \alpha \mathcal{U}(\lambda)}+\theta_{2}+\sum_{n=1}^{\infty} v_{n} \lambda^{-n}, \\
\mathcal{B}_{n \alpha}(\lambda) & =\lambda^{n} e^{i \alpha \mathcal{U}(\lambda)}+\sum_{n=1}^{\infty} f_{n \alpha, m+1} \lambda^{-m}
\end{aligned}
$$

where $g_{n}, u_{n}$ and $v_{n}$ are the same as in the definition of $\mathcal{M}, \mathcal{U}$ and $\mathcal{V}$. Siminarly, the coefficients of $\mathcal{B}_{n \alpha}(\lambda)$ are those of Laurent expansion of $\mathcal{B}_{n \alpha}$ in $\mathcal{L}$ :

$$
\mathcal{B}_{n \alpha}=\mathcal{L}^{n} e^{i \alpha \mathcal{U}(\lambda)}+\sum_{n=1}^{\infty} f_{n \alpha, m+1} \mathcal{L}^{-m}
$$

To see that $\mathcal{B}_{n \alpha}$ has such Laurent expansion, let us recall that $\mathcal{L}$ is a Laurent series of $k$ of the form

$$
\mathcal{L}=k+\sum_{n=1}^{\infty} g_{n+1}(t, x, \theta) k^{-n}
$$

This relation can be solved for $k$,

$$
k=\mathcal{L}+\sum_{n=1}^{\infty} f_{n+1}(t, x, \theta) \mathcal{L}^{-n} .
$$

(In the case of the dispersionless KP hierarchy, these coefficients $f_{n}$ are di-

rectly related with "flat coordinates" of topological Landau-Ginzburg models 
[6, 7, 8].) Since $\mathcal{B}_{n \alpha}$ is a polynomial in $k$, substitution of this expression of $k$ yields an expansion of $\mathcal{B}_{n \alpha}$ as mentioned above. Replacing $\mathcal{L} \rightarrow \lambda$, one obtains $\mathcal{B}_{n \alpha}(\lambda)$. In particular, since $\mathcal{B}_{10}=k, \mathcal{B}_{10}(\lambda)$ turns out to be written

$$
\mathcal{B}_{10}(\lambda)=\lambda+\sum_{n=1}^{\infty} f_{n+1} \lambda^{-n}
$$

Conversely, one can reproduce $\mathcal{L}, \mathcal{M}, \mathcal{U}$ and $\mathcal{V}$ from the above Laurent series of $\lambda$ by solving the relation

$$
k=\mathcal{B}_{10}(\lambda)
$$

for $\lambda$. this gives $\lambda=\mathcal{L}$ as a function of $(t, k, x, \theta)$. Substituting $\lambda=\mathcal{L}$ in $\mathcal{U}(\lambda), \mathcal{U}(\lambda)$ and $\mathcal{V}(\lambda)$ then give $\mathcal{M}, \mathcal{U}$ and $\mathcal{V}$.

The extended Lax system, too, can be converted into the language of the Latter Laurent series:

Proposition 2 Extended Lax system (3.4, 3.5) is equivalent to the following system consisting of three different sets of equations.

a) Lax equations

$$
\begin{aligned}
& \frac{\partial \mathcal{U}(\lambda)}{\partial t_{n \alpha}}=\left\{\mathcal{B}_{n \alpha}(\lambda), \mathcal{U}(\lambda)\right\}_{\theta}, \\
& \frac{\partial \mathcal{V}(\lambda)}{\partial t_{n \alpha}}=\left\{\mathcal{B}_{n \alpha}(\lambda), \mathcal{V}(\lambda)\right\}_{\theta}, \\
& \frac{\partial \mathcal{M}(\lambda)}{\partial t_{n \alpha}}=\frac{\partial \mathcal{B}_{n \alpha}(\lambda)}{\partial \lambda}+\left\{\mathcal{B}_{n \alpha}(\lambda), \mathcal{M}(\lambda)\right\}_{\theta},
\end{aligned}
$$

b) canonical relations (and related equations)

$$
\begin{aligned}
& \{\mathcal{U}(\lambda), \mathcal{V}(\lambda)\}_{\theta}=1, \\
& \{\mathcal{M}(\lambda), \mathcal{U}(\lambda)\}_{\theta}=\frac{\partial \mathcal{U}(\lambda)}{\partial \lambda}, \\
& \{\mathcal{M}(\lambda), \mathcal{V}(\lambda)\}_{\theta}=\frac{\partial \mathcal{V}(\lambda)}{\partial \lambda},
\end{aligned}
$$

c) zero-curvature equations

$$
\frac{\partial \mathcal{B}_{m \alpha}(\lambda)}{\partial t_{n \beta}}-\frac{\partial \mathcal{B}_{n \beta}(\lambda)}{\partial t_{m \alpha}}+\left\{\mathcal{B}_{m \alpha}(\lambda), \mathcal{B}_{n \beta}(\lambda)\right\}_{\theta}=0 .
$$

Here (3.49) is understood to include differential equations with respect to $x$, too, which are identical to the differential equations with respect to $t_{10}$. 
Remark Basically the same is also true for the planar model (except that the toroidal Poisson bracket $\{, \quad\}_{\theta}$ has to be replaced by the planar Poisson bracket $\{,\}_{y z}$ ). The dispersionless KP hierarchy, too, has a similar expression:

$$
\begin{aligned}
\frac{\partial \mathcal{M}(\lambda)}{\partial t_{n}} & =\frac{\partial \mathcal{B}_{n}(\lambda)}{\partial \lambda}, \\
\frac{\partial \mathcal{B}_{m}(\lambda)}{\partial t_{n}} & =\frac{\partial \mathcal{B}_{n}(\lambda)}{\partial t_{m}} .
\end{aligned}
$$

Note that this is a condition for the existence of a potential. Actually, the potential is given by the $S$ function $S(\lambda)$ [6, 7] i.e., the "phase function" of a quasi-classical (WKB) expression of the Baker-Akhiezer function [23, 16]. This is not the case of the higher dimensional hierarchy (because of the presence of the Poisson bracket terms in (3.42), indicating a crucial difference between those higher and lower dimensional integrable hierarchies.

\subsection{Proof of Proposition}

We here give a proof of Proposition 2. Since extended Lax system (3.4) is equivalent to 2 -form equation (3.20), it is sufficient to prove the equivalence with the 2 -form equation. Let us derive (3.42, 3.43, 3.44) from the 2 -form equation (3.20).

We first make a change of coordinates $(t, k, x, \theta) \rightarrow(t, \lambda, x, \theta)$ by

$$
\lambda=\mathcal{L}(k, x, \theta, t)
$$

Then 2-form equation (3.20) turns into the following form:

$d \lambda \wedge d \mathcal{M}(\lambda)+d \mathcal{U}(\lambda) \wedge d \mathcal{V}(\lambda)=d \mathcal{B}_{10}(\lambda) \wedge d x+d \theta_{1} \wedge d \theta_{2}+\sum_{n \alpha} d \mathcal{B}_{n \alpha}(\lambda) \wedge d t_{n \alpha}$

Both hand sides of this equation can be expanded into a linear combination of basic 2-forms: $d \theta_{1} \wedge d \theta_{2}, d \lambda \wedge d \theta_{j}, d \theta_{j} \wedge d t_{n \alpha}, d \lambda \wedge d t_{n \alpha}, d t_{m \alpha} \wedge d t_{n \beta}$, and those including $d x$. Relations among their coefficients will then give partial differential equations for $\mathcal{M}(\lambda), \mathcal{U}(\lambda)$ and $\mathcal{V}(\lambda)$. Since $\mathcal{B}_{10}=k, d x$ and $d t_{10}$ arise only as a linear combination of the form of $d x+d t_{10}$. Therefore 
differential equations including $\partial / \partial x$ should take the same form as those including $\partial / \partial t_{10}$; we do not have to consider them independently.

1. From $d \theta_{1} \wedge d \theta_{2}$,

$$
\frac{\partial(\mathcal{U}(\lambda), \mathcal{V}(\lambda))}{\partial\left(\theta_{1}, \theta_{2}\right)}=1
$$

This is exactly the first equation of (3.43).

2. From $d \lambda \wedge d \theta_{j}$,

$$
\frac{\partial \mathcal{M}(\lambda)}{\partial \theta_{j}}+\frac{\partial(\mathcal{U}(\lambda), \mathcal{V}(\lambda))}{\partial\left(\lambda, \theta_{j}\right)}=0
$$

These equations can be rewritten into a matrix form:

$$
\left(\frac{\partial \mathcal{V}(\lambda)}{\partial \lambda},-\frac{\partial \mathcal{U}(\lambda)}{\partial \lambda}\right)\left(\begin{array}{ll}
\frac{\partial \mathcal{U}(\lambda)}{\partial \theta_{1}} & \frac{\partial \mathcal{U}(\lambda)}{\partial \theta_{2}} \\
\frac{\partial \mathcal{V}(\lambda)}{\partial \theta_{1}} & \frac{\partial \mathcal{V}(\lambda)}{\partial \theta_{2}}
\end{array}\right)=\left(\frac{\partial \mathcal{M}(\lambda)}{\partial \theta_{1}}, \frac{\partial \mathcal{M}(\lambda)}{\partial \theta_{2}}\right)
$$

Since the determinant of the coefficient matrix coincides with $\{\mathcal{U}(\lambda), \mathcal{V}(\lambda)\}_{\theta}=$ 1 , these equations can be explicitly solved for $\partial \mathcal{U}(\lambda) / \partial \lambda$ and $\partial \mathcal{V}(\lambda) / \partial \lambda$ :

$$
\left(\frac{\partial \mathcal{V}(\lambda)}{\partial \lambda},-\frac{\partial \mathcal{U}(\lambda)}{\partial \lambda}\right)=\left(\frac{\partial \mathcal{M}(\lambda)}{\partial \theta_{1}}, \frac{\partial \mathcal{M}(\lambda)}{\partial \theta_{2}}\right)\left(\begin{array}{rr}
\frac{\partial \mathcal{V}(\lambda)}{\partial \theta_{2}} & -\frac{\partial \mathcal{U}(\lambda)}{\partial \theta_{2}} \\
-\frac{\partial \mathcal{V}(\lambda)}{\partial \theta_{1}} & \frac{\partial \mathcal{U}(\lambda)}{\partial \theta_{1}}
\end{array}\right)
$$

They give the second and third equations of (3.43).

3. From $d \theta_{j} \wedge d t_{n \alpha}$,

$$
\frac{\partial(\mathcal{U}(\lambda), \mathcal{V}(\lambda))}{\partial\left(\theta_{j}, t_{n \alpha}\right)}=\frac{\partial \mathcal{B}_{n \alpha}(\lambda)}{\partial \theta_{j}}
$$

These equation, too, can be solved for $\partial \mathcal{U}(\lambda) / \partial t_{n \alpha}$ and $\partial \mathcal{V}(\lambda) / \partial t_{n \alpha}$ in the same way. This gives the first and second equation of (3.42).

4. From $d \lambda \wedge d t_{n \alpha}$,

$$
\frac{\partial \mathcal{M}(\lambda)}{\partial t_{n \alpha}}+\frac{\partial(\mathcal{U}(\lambda), \mathcal{V}(\lambda))}{\partial\left(\lambda, t_{n \alpha}\right)}=\frac{\partial \mathcal{B}_{n \alpha}(\lambda)}{\partial \lambda}
$$


By using (3.42), which have been proven above, one can rewrite the second term on the left hand side as:

$$
\begin{aligned}
\frac{\partial(\mathcal{U}(\lambda), \mathcal{V}(\lambda))}{\partial\left(\lambda, t_{n \alpha}\right)}= & \{\mathcal{M}(\lambda), \mathcal{U}(\lambda)\}_{\theta}\left\{\mathcal{B}_{n \alpha}(\lambda), \mathcal{V}(\lambda)\right\}_{\theta} \\
& -\{\mathcal{M}(\lambda), \mathcal{V}(\lambda)\}_{\theta}\left\{\mathcal{B}_{n \alpha}(\lambda), \mathcal{V}(\lambda)\right\}_{\theta}
\end{aligned}
$$

and by (3.43), furthermore,

$$
=\left\{\mathcal{M}(\lambda), \mathcal{B}_{n \alpha}(\lambda)\right\}_{\theta}
$$

Thus the third equation of (3.42) follows.

5. From $d t_{m \alpha} \wedge d t_{n \beta}$,

$$
\frac{\partial(\mathcal{U}(\lambda), \mathcal{V}(\lambda))}{\partial\left(t_{m \alpha}, t_{n \alpha}\right)}=\frac{\partial \mathcal{B}_{n \beta}(\lambda)}{\partial t_{m \alpha}}-\frac{\partial \mathcal{B}_{m \alpha}(\lambda)}{\partial t_{n \beta}}
$$

The left hand side can be rewritten in the same way as above:

$$
\begin{aligned}
\frac{\partial(\mathcal{U}(\lambda), \mathcal{V}(\lambda))}{\partial\left(t_{m \alpha}, t_{n \alpha}\right)}= & \left\{\mathcal{B}_{m \alpha}(\lambda), \mathcal{U}(\lambda)\right\}_{\theta}\left\{\mathcal{B}_{n \beta}(\lambda), \mathcal{V}(\lambda)\right\}_{\theta} \\
& -\left\{\mathcal{B}_{m \alpha}(\lambda), \mathcal{V}(\lambda)\right\}_{\theta}\left\{\mathcal{B}_{n \beta}(\lambda), \mathcal{U}(\lambda)\right\}_{\theta} \\
= & \left\{\mathcal{B}_{m \alpha}(\lambda), \mathcal{B}_{n \beta}(\lambda)\right\}_{\theta}
\end{aligned}
$$

Thus (3.44) follows.

By carefully chasing these calculations, one will be able to show that the converse is also true.

\section{Symmetries in Lax formalism}

\subsection{Main results}

There are several different ways to obtain additional symmetries of this type of hierarchies. The most orthodox method is due to the twistor theoretical description of solutions [9]. The set of all twistor data $f$ forms a group of symplectic diffeomorphism, which acts on itself by left or right multiplications. Symmetries of the hierarchies are nothing but the action of this group

on the space of solutions induced by the correspondence between twistor data 
and solutions. Actually, this group action is highly nonlinear and difficut to describe in any explicit form. Its infinitesimal action, however, turns out to have a very explicit and simple form. This fact was pointed out first in the case of the self-dual Einstein equation [10], and has been verified for some other equations with a twistor theoretical structure.

Infinitesimal symmetries can also be obtained by directly solving their defining equations, such as (2.16) in the case of the dispersionless KP hierarchy. In the present case, these equations consist of the following:

(a) Four equations concerning Lax equations (3.4),

$$
\frac{\partial}{\partial t_{n \alpha}} \delta_{\mathcal{A}} \mathcal{L}=\left\{\delta_{\mathcal{A}} \mathcal{B}_{n \alpha}, \mathcal{L}\right\}+\left\{\mathcal{B}_{n \alpha}, \delta_{\mathcal{A}} \mathcal{L}\right\} \quad\left(=\delta_{\mathcal{A}} \frac{\partial \mathcal{L}}{\partial t_{n \alpha}}\right), \quad \text { etc. }
$$

(replacing $\mathcal{L} \rightarrow \mathcal{M}, \mathcal{U}, \mathcal{V}$ ), where $\delta_{\mathcal{A}} \mathcal{B}_{n \alpha}$ denotes the action on $\mathcal{B}_{n \alpha}$ induced by the relation $\mathcal{B}_{n \alpha}=\left(\mathcal{L}^{n} e^{i \alpha \mathcal{U}}\right)_{\geq 0}$. (These equations are understood to include the equations with respect to $x$, which are identical to the equations with respect to $t_{10}$.)

(b) Six equations concerning canonical relations (3.5),

$$
\left\{\delta_{\mathcal{A}} \mathcal{L}, \mathcal{M}\right\}+\left\{\mathcal{L}, \delta_{\mathcal{A}} \mathcal{M}\right\}=0, \quad \text { etc. }
$$

(replacing $\mathcal{L}, \mathcal{M} \rightarrow \mathcal{L}, \mathcal{M}, \mathcal{U}, \mathcal{V}$ ).

If one can solve these equations for $\delta \mathcal{L}$, etc. (and we shall indeed give a large set of such solutions below), the solutions give infinitesimal symmetries of the toroidal model. A very successful example of this kind of direct approach can be seen in the work of Park 24] on symmetries of the self-dual Einstein equations and related equations.

We now present our results on infinitesimal symmetries along the line of second approach. Actually, these results can also be derived from the twistor theoretical appoach, though we omit details. We shall further calculate commutation relations of those infinitesimal symmetries. Although the results are formulated in the framework of the toroidal model, basically the same construction can be applied to the planar model (and presumably to any other models).

Let $\mathcal{R}_{0}$ be the differential ring with generators $\left(g_{n}, h_{n}, u_{n}, v_{n}\right)$ and derivations $\left(\partial / \partial x, \partial / \partial \theta_{j}, \partial / \partial t_{n \alpha}\right)$, and $R$ the extension explicitly including the variables $\left(x, \theta_{j}, t_{n \alpha}\right)$. These differential rings represent the extended Lax system of the toroidal model. 
Theorem 1 Given an arbitrary Laurent-Fourier series of the form

$$
\mathcal{A}(\lambda, \mu, u, v)=\sum_{n=-\infty}^{\infty} \sum_{m=0}^{\infty} \sum_{\alpha, \beta=-\infty}^{\infty} \lambda^{n} \mu^{m} e^{i(\alpha u+\beta v)},
$$

let $\delta_{\mathcal{A}}$ denote a new derivation $\delta_{\mathcal{A}}: \mathcal{R} \rightarrow \mathcal{R}$ defined by

$$
\begin{aligned}
\delta_{\mathcal{A}} \mathcal{L} & =\left\{\mathcal{A}(\mathcal{L}, \mathcal{M}, \mathcal{U}, \mathcal{V})_{\leq-1}, \mathcal{L}\right\} \\
\delta_{\mathcal{A}} \mathcal{M} & =\left\{\mathcal{A}(\mathcal{L}, \mathcal{M}, \mathcal{U}, \mathcal{V})_{\leq-1}, \mathcal{M}\right\} \\
\delta_{\mathcal{A}} \mathcal{U} & =\left\{\mathcal{A}(\mathcal{L}, \mathcal{M}, \mathcal{U}, \mathcal{V})_{\leq-1}, \mathcal{U}\right\} \\
\delta_{\mathcal{A}} \mathcal{V} & =\left\{\mathcal{A}(\mathcal{L}, \mathcal{M}, \mathcal{U}, \mathcal{V})_{\leq-1}, \mathcal{V}\right\} \\
\delta_{\mathcal{A}} t_{n \alpha} & =\delta_{\mathcal{A}} x=\delta_{\mathcal{A}} \theta_{j}=1
\end{aligned}
$$

The four quantities $\left(\delta_{\mathcal{A}} \mathcal{L}, \delta_{\mathcal{A}} \mathcal{M}, \delta_{\mathcal{A}} \mathcal{U}, \delta_{\mathcal{A}} \mathcal{V}\right)$ satisfy (4.1) and (4.9), so that the derivation $\delta_{\mathcal{A}}$ gives a symmetry of the toroidal model.

Theorem 2 These symmetries obey the commutation relations

$$
\left[\delta_{\mathcal{A}}, \delta_{\mathcal{B}}\right]=\delta_{\{\mathcal{A}, \mathcal{B}\}_{\lambda \mu u v}}
$$

where $\mathcal{A}=\mathcal{A}(\lambda, \mu, u, v)$ and $\mathcal{B}=\mathcal{B}(\lambda, \mu, u, v)$ are two arbitrary LaurentFourier series as above, and the Poisson bracket of $\mathcal{A}$ and $\mathcal{B}$ is defined by

$$
\{\mathcal{A}, \mathcal{B}\}_{\lambda \mu u v}=\frac{\partial \mathcal{A}}{\partial \lambda} \frac{\partial \mathcal{B}}{\partial \mu}-\frac{\partial \mathcal{A}}{\partial \mu} \frac{\partial \mathcal{B}}{\partial \lambda}+\frac{\partial \mathcal{A}}{\partial u} \frac{\partial \mathcal{B}}{\partial v}-\frac{\partial \mathcal{A}}{\partial v} \frac{\partial \mathcal{B}}{\partial u} .
$$

Proof of these results are presented in the next two subsections.

\subsection{Proof of the first theorem}

We here prove Theorem 1. To simplify notations, let $\mathcal{A}$ denote $\mathcal{A}(\mathcal{L}, \mathcal{M}, \mathcal{U}, \mathcal{V})$ in the following. We have to show that (4.1) and (4.2) are indeed satisfied.

Let us examine (4.1). By the defining equation of $\delta_{\mathcal{A}} \mathcal{L}$ and the Lax equations, the left hand side of (4.1) can be calculated as:

$$
\begin{aligned}
\text { LHS of (4.1) } & =\frac{\partial}{\partial t_{n \alpha}}\left\{\mathcal{A}_{\leq-1}, \mathcal{L}\right\} \\
& =\left\{\frac{\partial \mathcal{A}_{\leq-1}}{\partial t_{n \alpha}}, \mathcal{L}\right\}+\left\{\mathcal{A}_{\leq-1}, \frac{\partial \mathcal{L}}{\partial t_{n \alpha}}\right\} \\
& =\left\{\frac{\partial \mathcal{A}_{\leq-1}}{\partial t_{n \alpha}}, \mathcal{L}\right\}+\left\{\mathcal{A}_{\leq-1},\left\{\mathcal{B}_{n \alpha}, \mathcal{L}\right\}\right\}
\end{aligned}
$$


Similarly,

$$
\operatorname{RHS} \text { of }(4.1)=\left\{\delta_{\mathcal{A}} \mathcal{B}_{n \alpha}, \mathcal{L}\right\}+\left\{\mathcal{B}_{n \alpha},\left\{\mathcal{A}_{\leq-1}, \mathcal{B}_{n \alpha}\right\}\right\}
$$

Subtracting the former from the latter and using the Jacobi identity for the Poisson bracket, one can show that

$$
\operatorname{LHS}-\operatorname{RHS} \text { of }(4.1)=\left\{\frac{\partial \mathcal{A}_{\leq-1}}{\partial t_{n \alpha}}-\delta_{\mathcal{A}} \mathcal{B}_{n \alpha}+\left\{\mathcal{A}_{\leq-1}, \mathcal{B}_{n \alpha}\right\}, \mathcal{L}\right\}
$$

We now argue that

$$
\frac{\partial \mathcal{A}_{\leq-1}}{\partial t_{n \alpha}}-\delta_{\mathcal{A}} \mathcal{B}_{n \alpha}+\left\{\mathcal{A}_{\leq-1}, \mathcal{B}_{n \alpha}\right\}=0
$$

To show this relation, let us note the following obvious consequence of the Lax equations and the Leibniz rule satisfied by $\left\{\mathcal{B}_{n \alpha}, \cdot\right\}$ :

$$
\frac{\partial \mathcal{A}}{\partial t_{n \alpha}}=\left\{\mathcal{B}_{n \alpha}, \mathcal{A}\right\}
$$

The ()$_{\leq-1}$ part of this identity gives

$$
\frac{\partial \mathcal{A}_{\leq-1}}{\partial t_{n \alpha}}=\left(\left\{\mathcal{B}_{n \alpha}, \mathcal{A}_{\leq-1}\right\}\right)_{\leq-1}
$$

Similarly, by the defining equation of $\delta_{\mathcal{A}}$ and the Leibniz rule satisfied by $\left\{\mathcal{A}_{\leq-1}, \cdot\right\}$

$$
\delta_{\mathcal{A}}\left(\mathcal{L}^{n} e^{i \alpha \mathcal{U}}\right)=\left\{\mathcal{A}_{\leq-1}, \mathcal{L}^{n} e^{i \alpha \mathcal{U}}\right\}
$$

The ()$_{\geq 0}$ part of both hand sides gives

$$
\delta_{\mathcal{A}} \mathcal{B}_{n \alpha}=\left(\left\{\mathcal{A}_{\leq-1}, \mathcal{B}_{n \alpha}\right\}\right)_{\geq 0}
$$

Combining these two equations, one finds that

$$
\begin{aligned}
\frac{\partial \mathcal{A}_{\leq-1}}{\partial t_{n \alpha}}-\delta_{\mathcal{A}} \mathcal{B}_{n \alpha} & =\left(\left\{\mathcal{B}_{n \alpha}, \mathcal{A}_{\leq-1}\right\}\right)_{\leq-1}+\left(\left\{\mathcal{A}_{\leq-1}, \mathcal{B}_{n \alpha}\right\}\right)_{\geq 0} \\
& =\left\{\mathcal{B}_{n \alpha}, \mathcal{A}_{\leq-1}\right\}
\end{aligned}
$$

which is exactly the relation that we wish to derive. 
Thus the right hand side of (4.9) turns out to vanish, and (4.1) is proven for $\delta_{\mathcal{A}} \mathcal{L}$. Other equations of (4.1), too, can be checked by the same way.

We next consider (4.2). They are rather immediate from the Jacobi identities. For instance,

$$
\begin{aligned}
\left\{\delta_{\mathcal{A}} \mathcal{L}, \mathcal{M}\right\}+\left\{\mathcal{L}, \delta_{\mathcal{A}} \mathcal{M}\right\} & =\left\{\left\{\mathcal{A}_{\leq-1}, \mathcal{L}\right\}, \mathcal{M}\right\}+\left\{\mathcal{L},\left\{\mathcal{A}_{\leq-1}, \mathcal{M}\right\}\right\} \\
& =\left\{\mathcal{A}_{\leq-1},\{\mathcal{L}, \mathcal{M}\}\right\} \\
& =\left\{\mathcal{A}_{\leq-1}, 1\right\} \\
& =0
\end{aligned}
$$

The other equations of (4.2) can be derived in the same way.

\subsection{Proof of the second theorem}

We now prove Theorem 2. Also here, $\mathcal{A}$ and $\mathcal{B}$ denote $\mathcal{A}(\mathcal{L}, \mathcal{M}, \mathcal{U}, \mathcal{V})$ and $\mathcal{B}(\mathcal{L}, \mathcal{M}, \mathcal{U}, \mathcal{V})$. As we shall show below, the proof is almost parallel to the proof Theorem 11 .

First examine the difference of both hand side of (4.5). One can calculate the repeated action $\delta_{\mathcal{A}} \delta_{\mathcal{B}} \mathcal{L}$ of two symmetries just as in the calculation of the right hand side of (4.1):

$$
\begin{aligned}
\delta_{\mathcal{A}} \delta_{\mathcal{B}} \mathcal{L} & =\delta_{\mathcal{A}}\left\{\mathcal{B}_{\leq-1}, \mathcal{L}\right\} \\
& =\left\{\delta_{\mathcal{A}} \mathcal{B}_{\leq-1}, \mathcal{L}\right\}+\left\{\mathcal{B}_{\leq-1}, \delta_{\mathcal{A}} \mathcal{L}\right\} \\
& =\left\{\delta_{\leq-1}, \mathcal{L}\right\}+\left\{\mathcal{B}_{\leq-1},\{\mathcal{A}, \mathcal{L}\}\right\}
\end{aligned}
$$

Subtracting from this the same equation with $\mathcal{A}$ and $\mathcal{B}$ interchanged, and using the Jacobi identity, one can derive

$$
\left[\delta_{\mathcal{A}}, \delta_{\mathcal{B}}\right] \mathcal{L}=\left\{\delta_{\mathcal{A}} \mathcal{B}_{\leq-1}-\delta_{\mathcal{B}} \mathcal{A}_{\leq-1}+\left\{\mathcal{B}_{\leq-1}, \mathcal{A}_{\leq-1}\right\}, \mathcal{L}\right\}
$$

Meanwhile, by canonical relations (3.5), one can easily show that

$$
\{\mathcal{A}, \mathcal{B}\}=\left.\{\mathcal{A}(\lambda, \mu, u, v), \mathcal{B}(\lambda, \mu, u, v)\}_{\lambda \mu u v}\right|_{\lambda=\mathcal{L}, \mu=\mathcal{M}, u=\mathcal{U}, v=\mathcal{V}} .
$$

Combining this with the above identity, one obtains the relation

$$
\begin{aligned}
& {\left[\delta_{\mathcal{A}}, \delta_{\mathcal{B}}\right] \mathcal{L}-\delta_{\{\mathcal{A}, \mathcal{B}\}_{\lambda \mu u v}} \mathcal{L} } \\
= & \left\{\delta_{\mathcal{A}} \mathcal{B}_{\leq-1}-\delta_{\mathcal{B}} \mathcal{A}_{\leq-1}+\left\{\mathcal{B}_{\leq-1}, \mathcal{A}_{\leq-1}\right\}-\left(\{\mathcal{A}, \mathcal{B}\}_{\lambda \mu u v}\right)_{\leq-1}, \mathcal{L}\right\}
\end{aligned}
$$


We now argue that

$$
\delta_{\mathcal{A}} \mathcal{B}_{\leq-1}-\delta_{\mathcal{B}} \mathcal{A}_{\leq-1}+\left\{\mathcal{B}_{\leq-1}, \mathcal{A}_{\leq-1}\right\}-\left(\{\mathcal{A}, \mathcal{B}\}_{\lambda \mu u v}\right)_{\leq-1}=0
$$

To show this, we now start from the identities

$$
\delta_{\mathcal{A}} \mathcal{B}=\left\{\mathcal{A}_{\leq-1}, \mathcal{B}\right\}, \quad \delta_{\mathcal{B}} \mathcal{A}=\left\{\mathcal{B}_{\leq-1}, \mathcal{A}\right\},
$$

which follow from the definition of $\delta_{\mathcal{A}}$ and $\delta_{\mathcal{B}}$ and the Leibniz rule satisfied by $\delta_{\mathcal{A}}, \delta_{\mathcal{B}},\left\{\mathcal{A}_{\leq-1}, \cdot\right\}$ and $\left\{\mathcal{B}_{\leq-1}, \cdot\right\}$. Now one can use the same method of calculations as employed in the proof of Theorem 1 to show that

$$
\begin{aligned}
& \delta_{\mathcal{A}} \mathcal{B}_{\leq-1}-\delta_{\mathcal{B}} \mathcal{A}_{\leq-1}-\left\{\mathcal{A}_{\leq-1}, \mathcal{B}_{\leq-1}\right\}-(\{\mathcal{A}, \mathcal{B}\})_{\leq-1} \\
= & \left(\left\{\mathcal{A}_{\leq-1}, \mathcal{B}\right\}-\left\{\mathcal{B}_{\leq-1}, \mathcal{A}\right\}-\left\{\mathcal{A}_{\leq-1}, \mathcal{B}_{\leq-1}\right\}-\{\mathcal{A}, \mathcal{B}\}\right)_{\leq-1}
\end{aligned}
$$

and further substituting $\mathcal{A}=\mathcal{A}_{\leq-1}+\mathcal{A}_{\geq 0}$ etc.,

$$
\begin{aligned}
= & \left(\left\{\mathcal{A}_{\leq-1}, \mathcal{B}_{\leq-1}\right\}+\left\{\mathcal{A}_{\leq-1}, \mathcal{B}_{\geq 0}\right\}-\left\{\mathcal{B}_{\leq-1}, \mathcal{A}_{\leq-1}\right\}-\left\{\mathcal{B}_{\leq-1}, \mathcal{A}_{\geq 0}\right\}\right. \\
& -\left\{\mathcal{A}_{\leq-1}, \mathcal{B}_{\leq-1}\right\} \\
& \left.-\left\{\mathcal{A}_{\leq-1}, \mathcal{B}_{\leq-1}\right\}-\left\{\mathcal{A}_{\leq-1}, \mathcal{B}_{\geq 0}\right\}-\left\{\mathcal{A}_{\geq 0}, \mathcal{B}_{\leq-1}\right\}-\left\{\mathcal{A}_{\geq 0}, \mathcal{B}_{\geq 0}\right\}\right)_{\leq-1} \\
= & 0
\end{aligned}
$$

This is the relation that we wish to derive, which implies that (4.2) is satisfied by $\mathcal{L}$ and $\mathcal{M}$.

The other conditions of (4.2), too, can be similarly checked.

\subsection{Another expression of symmetries}

The following result, which we shall use later, shows what the action of infinitesimal symmetries look like in terms of the the Laurent series $\mathcal{M}(\lambda)$, $\mathcal{U}(\lambda)$ and $\mathcal{V}(\lambda)$

Theorem 3 The symmetries $\delta_{\mathcal{A}}$ act on $\mathcal{M}(\lambda), \mathcal{U}(\lambda)$ and $\mathcal{V}(\lambda)$ as:

$$
\begin{aligned}
\delta_{\mathcal{A}} \mathcal{M}(\lambda) & =\frac{\partial \mathcal{A}_{\leq-1}(\lambda)}{\partial \lambda}+\left\{\mathcal{A}_{\leq-1}(\lambda), \mathcal{M}(\lambda)\right\}_{\theta} \\
\delta_{\mathcal{A}} \mathcal{U}(\lambda) & =\left\{\mathcal{A}_{\leq-1}(\lambda), \mathcal{U}(\lambda)\right\}_{\theta} \\
\delta_{\mathcal{A}} \mathcal{V}(\lambda) & =\left\{\mathcal{A}_{\leq-1}(\lambda), \mathcal{V}(\lambda)\right\}_{\theta}
\end{aligned}
$$


where $\mathcal{A}_{\leq-1}(\lambda)$ is the Laurent series obtained by expanding $\mathcal{A}_{\leq-1}$ into Laurent series of $\mathcal{L}$,

$$
\mathcal{A}_{\leq-1}=\sum_{n=-\infty}^{\infty} a_{n}(t, x, \theta) \mathcal{L}^{n}
$$

and replacing $\mathcal{L} \rightarrow \lambda$,

$$
\mathcal{A}_{\leq-1}(\lambda)=\sum_{n=-\infty}^{\infty} a_{n}(t, x, \theta) \lambda^{n}
$$

Proof Let us first derive the formula for $\mathcal{M}(\lambda)$. Starting with the obvious identity

$$
\delta_{\mathcal{A}} \mathcal{M}=\left.\delta_{\mathcal{A}} \mathcal{M}(\lambda)\right|_{\lambda=\mathcal{L}}+\left.\frac{\partial \mathcal{M}(\lambda)}{\partial \lambda}\right|_{\lambda=\mathcal{L}} \delta_{\mathcal{A}} \mathcal{L}
$$

we now calculate both hand sides as follows. First, by the Leibniz rule satisfied by $\{\cdot, \mathcal{L}\}$,

$$
\begin{aligned}
\delta_{\mathcal{A}} \mathcal{L} & =\left\{\mathcal{A}_{\leq-1}, \mathcal{L}\right\} \\
& =\left.\left\{\mathcal{A}_{\leq-1}(\lambda), \mathcal{L}\right\}\right|_{\lambda=\mathcal{L}}+\left.\frac{\partial \mathcal{A}_{\leq-1}(\lambda)}{\partial \lambda}\right|_{\lambda=\mathcal{L}}\{\mathcal{L}, \mathcal{L}\}
\end{aligned}
$$

and since $\{\mathcal{L}, \mathcal{L}\}=0$, the right hand side of (4.27) can be rewritten

$$
\operatorname{RHS} \text { of }(4.27)=\left.\delta_{\mathcal{A}} \mathcal{M}(\lambda)\right|_{\lambda=\mathcal{L}}+\left.\frac{\partial \mathcal{M}(\lambda)}{\partial \lambda}\left\{\mathcal{A}_{\leq-1}(\lambda), \mathcal{L}\right\}\right|_{\lambda=\mathcal{L}} .
$$

Meanwhile, by the Leibniz rule satisfied by $\{\cdot, \mathcal{M}\}$, similarly,

$$
\begin{aligned}
\delta_{\mathcal{A}} \mathcal{M} & =\left\{\mathcal{A}_{\leq-1}, \mathcal{M}\right\} \\
& =\left.\left\{\mathcal{A}_{\leq-1}(\lambda), \mathcal{M}\right\}\right|_{\lambda=\mathcal{L}}+\left.\frac{\partial \mathcal{A}_{\leq-1}(\lambda)}{\partial \lambda}\right|_{\lambda=\mathcal{L}}\{\mathcal{L}, \mathcal{M}\} .
\end{aligned}
$$

Since $\{\mathcal{L}, \mathcal{M}\}=1$, and the Leibniz rule satisfied by $\left\{\mathcal{A}_{\leq-1}(\lambda), \cdot\right\}$ implies the identity

$$
\left\{\mathcal{A}_{\leq-1}(\lambda), \mathcal{M}\right\}=\left.\left\{\mathcal{A}_{\leq-1}(\lambda), \mathcal{M}(\lambda)\right\}\right|_{\lambda=\mathcal{L}}+\left.\frac{\partial \mathcal{M}(\lambda)}{\partial \lambda}\right|_{\lambda=\mathcal{L}}\left\{\mathcal{A}_{\leq-1}(\lambda), \mathcal{L}\right\}
$$


we now obtain the following expression for the left hand side of (4.27):

$$
\begin{aligned}
\text { LHS of }(4.27)= & \left.\left\{\mathcal{A}_{\leq-1}(\lambda), \mathcal{M}(\lambda)\right\}\right|_{\lambda=\mathcal{L}}+\left.\frac{\partial \mathcal{M}(\lambda)}{\partial \lambda}\left\{\mathcal{A}_{\leq-1}(\lambda), \mathcal{L}\right\}\right|_{\lambda=\mathcal{L}} \\
& +\left.\frac{\partial \mathcal{A}_{\leq-1}(\lambda)}{\partial \lambda}\right|_{\lambda=\mathcal{L}} .
\end{aligned}
$$

Plugging these relations into 4.27$)$ gives

$$
\left.\delta_{\mathcal{A}} \mathcal{M}(\lambda)\right|_{\lambda=\mathcal{L}}=\left.\frac{\partial \mathcal{A}_{\leq-1}(\lambda)}{\partial \lambda}\right|_{\lambda=\mathcal{L}}+\left.\left\{\mathcal{A}_{\leq-1}(\lambda), \mathcal{M}(\lambda)\right\}\right|_{\lambda=\mathcal{L}}
$$

We can now remove " $\lambda=\mathcal{L}$ " to obtain

$$
\delta_{\mathcal{A}} \mathcal{M}(\lambda)=\frac{\partial \mathcal{A}_{\leq-1}(\lambda)}{\partial \lambda}+\left\{\mathcal{A}_{\leq-1}(\lambda), \mathcal{M}(\lambda)\right\}
$$

The last Poisson bracket can be replaced by $\{\quad, \quad\}_{\theta}$ because both $\mathcal{A}_{\leq-1}(\lambda)$ and $\mathcal{M}(\lambda)$ do not depend on $k$. The first formula of (4.24) is thus proven.

The formulas for $\mathcal{U}(\lambda)$ and $\mathcal{V}(\lambda)$, too, can be derived in exactly the same way. Now start from the identity

$$
\delta_{\mathcal{A}} \mathcal{U}=\left.\delta_{\mathcal{A}} \mathcal{U}(\lambda)\right|_{\lambda=\mathcal{L}}+\left.\frac{\partial \mathcal{U}(\lambda)}{\partial \lambda}\right|_{\lambda=\mathcal{L}} \delta_{\mathcal{A}} \mathcal{L}
$$

and calculate both hand sides. The right hand side can be calculated as:

$$
\begin{aligned}
\text { RHS of (4.35) } & =\left.\delta_{\mathcal{A}} \mathcal{U}(\lambda)\right|_{\lambda=\mathcal{L}}+\left.\frac{\partial \mathcal{U}(\lambda)}{\partial \lambda}\left\{\mathcal{A}_{\leq-1}, \mathcal{L}\right\}\right|_{\lambda=\mathcal{L}} \\
& =\left.\delta_{\mathcal{A}} \mathcal{U}(\lambda)\right|_{\lambda=\mathcal{L}}+\left.\frac{\partial \mathcal{U}(\lambda)}{\partial \lambda}\left\{\mathcal{A}_{\leq-1}(\lambda), \mathcal{L}\right\}\right|_{\lambda=\mathcal{L}} .
\end{aligned}
$$

Similarly,

$$
\begin{aligned}
\operatorname{LHS} \text { of }(4.35) & =\left\{\mathcal{A}_{\leq-1}, \mathcal{U}\right\} \\
& =\left.\left\{\mathcal{A}_{\leq-1}(\lambda), \mathcal{U}\right\}\right|_{\lambda=\mathcal{L}}+\left.\left\{\mathcal{A}_{\leq-1}(\lambda), \mathcal{L}\right\}\right|_{\lambda=\mathcal{L}}\{\mathcal{L}, \mathcal{U}\} \\
& =\left.\left\{\mathcal{A}_{\leq-1}(\lambda), \mathcal{U}(\lambda)\right\}\right|_{\lambda=\mathcal{L}}+\left.\left\{\mathcal{A}_{\leq-1}(\lambda), \mathcal{L}\right\} \frac{\partial \mathcal{U}(\lambda)}{\partial \lambda}\right|_{\lambda=\mathcal{L}}(4.3
\end{aligned}
$$


Combining these relations, we now obtain

$$
\left.\delta_{\mathcal{A}} \mathcal{U}(\lambda)\right|_{\lambda=\mathcal{L}}=\left.\left\{\mathcal{A}_{\leq-1}(\lambda), \mathcal{U}(\lambda)\right\}\right|_{\lambda=\mathcal{L}}
$$

Again " $\lambda=\mathcal{L}$ " can be removed, and the Poisson bracket in the resulting equation may be replaced by $\{,\}_{\theta}$, so that we can derive the formula for $\mathcal{U}(\lambda)$. The formula for $\mathcal{V}(\lambda)$ can be treated in the same way.

\section{$5 \quad F$ function and symmetries}

\subsection{Main results}

We here introduce an analogue of the $F$ function for the toroidal model of higher dimensional dispersionless hierarchy, and extend the infinite dimensional symmetries of the last section to this $F$ function. The extended symmetries turn out to obey anomalous commutation relations.

The $F$ function of the dispersionless KP hierarchy is defined by (2.22) (or by and (2.23)). Note that these equations can be rewritten

$$
\begin{aligned}
& \frac{\partial F}{\partial t_{n}}=\operatorname{res}_{\lambda} \lambda^{n} \mathcal{M}(\lambda) \quad(n=1,2, \ldots), \\
& \frac{\partial F}{\partial x}=\operatorname{res}_{\lambda} \lambda \mathcal{M}(\lambda) .
\end{aligned}
$$

The following theorem shows a similar result for the toroidal model.

Theorem 4 Suppose that for any $\lambda$ in a neighborhood of $\infty$, the symplectic map $\theta \mapsto(\mathcal{U}(\theta), \mathcal{V}(\theta))$ is a diffeomorphism of the torus onto itself. The following system of equations is then integrable in the sense of Frobenius, and hence defines a function $F=F(t, x)$ of $(t, x)$ up to an integration constant:

$$
\begin{aligned}
\frac{\partial F}{\partial t_{n \alpha}} & =\int \frac{d \theta_{1} d \theta_{2}}{(2 \pi)^{2}} \underset{\lambda}{\operatorname{res}} \lambda^{n} \mathcal{M}(\lambda) e^{i \alpha \mathcal{U}(\lambda)}, \\
\frac{\partial F}{\partial x} & =\int \frac{d \theta_{1} d \theta_{2}}{(2 \pi)^{2}} \underset{\lambda}{\operatorname{res}} \lambda \mathcal{M}(\lambda), \\
\frac{\partial F}{\partial \theta_{j}} & =0
\end{aligned}
$$

where the integrals with respect to $\theta$ are over the torus $T^{2}\left(0 \leq \theta_{j} \leq 2 \pi\right)$. 
Remark 1. Since the right hand side of the equations of $\partial F / \partial t_{10}$ and $\partial F / \partial x$ are the same, the $F$ function, like other quantities, depends on $t_{10}$ and $x$ only through the linear combination $t_{10}+x$.

2. The $F$ function of the dispersionless dKP hierarchy can reproduce all other quantities $\left(g_{n}, h_{n}\right.$, etc.) by differentiation. Because of this, the whole hierarchy, in principle, can be converted into a system of differential equations for $F$. This is not the case of the toroidal model (and presumably of other higher dimensional integrable hierarchies).

To formulate results on symmetries, let us recall the language of differential rings. Following the case of the dispersionless KP hierarchy, we enlarge the rings $\mathcal{R}_{0}$ and $\mathcal{R}$ into $\mathcal{R}_{0}[F]$ and $\mathcal{R}[F]$. Eqs. (5.2) are then considered to be defining derivations $\partial / \partial t_{n \alpha}, \partial / \partial x$ and $\partial / \partial \theta_{j}$ on these rings rather than defining $F$ itself, and the theorem asserts that this extension of derivations is well defined.

Theorem 5 The symmetries $\delta_{\mathcal{A}}$ on $\mathcal{R}$ can be extended to symmetries on $\mathcal{R}[F]$ by

$$
\delta_{\mathcal{A}} F=-\int \frac{d \theta_{1} d \theta_{2}}{(2 \pi)^{2}} \operatorname{res}_{\lambda} \int_{0}^{\mathcal{M}(\lambda)} d \mu \mathcal{A}(\lambda, \mu, \mathcal{U}(\lambda), \mathcal{V}(\lambda)) .
$$

Namely, this defines a new additional derivation on $\mathcal{R}[F]$ that commutes with $\partial / \partial t_{n \alpha}, \partial / \partial x$ and $\partial / \partial \theta_{j}$.

Theorem 6 Under the same assumption as Theorem $\mathbf{4}$, these symmetries on $\mathcal{R}[F]$ obey the anomalous commutation relations

$$
\left[\delta_{\mathcal{A}}, \delta_{\mathcal{B}}\right]=\delta_{\{\mathcal{A}, \mathcal{B}\}_{\lambda \mu u v}}+c(\mathcal{A}, \mathcal{B}) \partial_{F},
$$

where $\{, \quad\}_{\lambda \mu u v}$ is the same four dimensional Poisson bracket as in Theorem $1, c(\mathcal{A}, \mathcal{B})$ is a cocycle of the four dimensional Poisson algebra given by

$$
c(\mathcal{A}, \mathcal{B})=\int \frac{d u d v}{(2 \pi)^{2}} \operatorname{res}_{\lambda} \mathcal{A}(\lambda, 0, u, v) \frac{\partial}{\partial \lambda} \mathcal{B}(\lambda, 0, u, v),
$$

and $\partial_{F}$ is another derivation on $\mathcal{R}[F]$ uniquely determined by

$$
\partial_{F}(F)=1, \quad \partial_{F}(\text { any other generator of } \mathcal{R})=0 .
$$


Thus the commutation relations of extended symmetries obet the structure of a one-dimensional central extension of the four dimensional Poisson algebra with the Poisson bracket $\{\mathcal{A}, \mathcal{B}\}_{\lambda \mu u v}$. This result, too, is a very natural extension of the corresponding result in the dispersionless KP hierarchy.

The proofs of these theorems are more or less similar to each other. We first present the proof of Theorem 1 in some detail, and then organize the others in much the same, but slightly compressed form.

\subsection{Proof of the first theorem}

We now prove Theorem 1 . Let $h_{n \alpha} h_{10}$ denote the right hand side of the first equation of (5.2):

$$
h_{n \alpha}=\int \frac{d \theta_{1} d \theta_{2}}{(2 \pi)^{2}} \operatorname{res}_{\lambda} \lambda^{n} \mathcal{M}(\lambda) e^{i \alpha \mathcal{U}(\lambda)} .
$$

The Frobenius integrability condition for these quantities are given by

$$
\frac{\partial h_{n \beta}}{\partial t_{m \alpha}}=\frac{\partial h_{m \alpha}}{\partial t_{n \beta}} .
$$

We have only to chech these conditions. The others concerning the $x$ derivatives are identical to those of $t_{10}$-derivatives, and included in the above conditions as a special case.

1. Let us calculate $\partial h_{n \beta} / \partial t_{m \alpha}$. First, differentiating the above defining equation of $h_{n \beta}$, we have

$$
\frac{\partial h_{n \beta}}{\partial t_{m \alpha}}=\int \frac{d \theta_{1} d \theta_{2}}{(2 \pi)^{2}} \operatorname{res}_{\lambda} \lambda^{n}\left(\frac{\partial e^{i \beta \mathcal{U}(\lambda)}}{\partial t_{m \alpha}} \mathcal{M}(\lambda)+e^{i \beta \mathcal{U}(\lambda)} \frac{\partial \mathcal{M}(\lambda)}{\partial t_{m \alpha}}\right),
$$

and plugging Lax equations (3.42) for $\mathcal{M}(\lambda)$ and $\mathcal{U}(\lambda)$,

$$
\begin{aligned}
= & \int \frac{d \theta_{1} d \theta_{2}}{(2 \pi)^{2}} \operatorname{res}_{\lambda} \lambda^{n}\left\{\mathcal{B}_{m \alpha}(\lambda), e^{i \beta \mathcal{U}(\lambda)}\right\}_{\theta} \mathcal{M}(\lambda) \\
& +\int \frac{d \theta_{1} d \theta_{2}}{(2 \pi)^{2}} \underset{\lambda}{\operatorname{res}} \lambda^{n} e^{i \beta \mathcal{U}(\lambda)}\left(\frac{\partial \mathcal{B}_{m \alpha}(\lambda)}{\partial \lambda}+\left\{\mathcal{B}_{m \alpha}(\lambda), \mathcal{M}(\lambda)\right\}_{\theta}\right) \\
= & \int \frac{d \theta_{1} d \theta_{2}}{(2 \pi)^{2}} \operatorname{res}_{\lambda} \lambda^{n}\left\{\mathcal{B}_{m \alpha}(\lambda), e^{i \beta \mathcal{U}(\lambda)} \mathcal{M}(\lambda)\right\}_{\theta} \\
& +\int \frac{d \theta_{1} d \theta_{2}}{(2 \pi)^{2}} \operatorname{res}_{\lambda} \lambda^{n} e^{i \beta \mathcal{U}(\lambda)} \frac{\partial \mathcal{B}_{m \alpha}(\lambda)}{\partial \lambda} .
\end{aligned}
$$


Let us examine the first and second terms in the last two lines:

(i) The first term turns out vanish because of the following lemma.

Lemma 1 For any two functions $A=A(\theta)$ and $B=B(\theta)$ on the torus,

$$
\int \frac{d \theta_{1} d \theta_{2}}{(2 \pi)^{2}}\{A, B\}_{\theta}=0 .
$$

Proof The Poisson bracket can be re written into a total derivative:

$$
\{A, B\}_{\theta}=\frac{1}{2} \frac{\partial}{\partial \theta_{2}}\left(\frac{\partial A}{\partial \theta_{1}}-A \frac{\partial B}{\partial \theta_{1}}\right)-\frac{1}{2} \frac{\partial}{\partial \theta_{1}}\left(\frac{\partial A}{\partial \theta_{2}}-A \frac{\partial B}{\partial \theta_{2}}\right) .
$$

(ii) To handle the second term, recall the relation between $\mathcal{B}_{m \alpha}$ and $\mathcal{B}_{m \alpha}(\lambda)$. Since $\mathcal{B}_{m \alpha}$ depends on $k$ only through $\lambda=\mathcal{L}(t, x, \theta, k)$,

$$
\frac{\partial \mathcal{B}_{m \alpha}}{\partial k}=\left.\frac{\partial \mathcal{B}_{m \alpha}(\lambda)}{\partial \lambda}\right|_{\lambda=\mathcal{L}} \frac{\partial \mathcal{L}}{\partial k} .
$$

By this relation and the following lemma, we can rewrite the $\lambda$-residue into a $k$-residue:

$$
\begin{aligned}
\operatorname{res}_{\lambda} \lambda^{n} e^{i \beta \mathcal{U}(\lambda)} \frac{\partial \mathcal{B}_{m \alpha}(\lambda)}{\partial \lambda} & =\left.\operatorname{res}_{k} \mathcal{L}^{n} e^{i \beta \mathcal{U}} \frac{\partial \mathcal{B}_{m \alpha}(\lambda)}{\partial \lambda}\right|_{\lambda=\mathcal{L}} \frac{\partial \mathcal{L}}{\partial k} \\
& =\operatorname{res}_{k} \mathcal{L}^{n} e^{i \beta \mathcal{U}} \frac{\partial \mathcal{B}_{m \alpha}}{\partial k} .
\end{aligned}
$$

Lemma 2 Under a change of coordinates at $\infty$ of the form $\lambda=\mathcal{L}(k)=$ $k+O\left(k^{-1}\right)$, the residues with respect to $\lambda$ and $k$ are connected as

$$
\underset{\lambda}{\operatorname{res}} f(\lambda)=\operatorname{res}_{k} f(\mathcal{L}) \frac{\partial \mathcal{L}}{\partial k} .
$$

Proof Since the residue can be written as a contour integral (along a small loop encircling $\infty$ ),

$$
\underset{\lambda}{\operatorname{res}} f(\lambda)=\oint \frac{d \lambda}{2 \pi i} f(\lambda)
$$


the above formula is nothing but the formula of change of variables of integrals, $\partial \mathcal{L} / \partial k$ being the Jacobian.

Plugging these into the previous expression of $\partial h_{n \beta} / \partial t_{m \alpha}$, we arrive at the following intermediate result:

$$
\begin{aligned}
\frac{\partial h_{n \beta}}{\partial t_{m \alpha}} & =\int \frac{d \theta_{1} d \theta_{2}}{(2 \pi)^{2}} \operatorname{res}_{k} \mathcal{L}^{n} e^{i \beta \mathcal{U}} \frac{\partial}{\partial k} \mathcal{B}_{m \alpha} \\
& =\int \frac{d \theta_{1} d \theta_{2}}{(2 \pi)^{2}} \operatorname{res}_{k}\left(\mathcal{L}^{n} e^{i \beta \mathcal{U}}\right)_{\leq-1} \frac{\partial}{\partial k}\left(\mathcal{L}^{m} e^{i \alpha \mathcal{U}}\right)_{\geq 0} .
\end{aligned}
$$

2. We now subtract from the last equation the same equation with $(m, \alpha)$ and $(n, \beta)$ interchanged. This gives

$$
\begin{gathered}
\frac{\partial h_{n \beta}}{\partial t_{m \alpha}}-\frac{\partial h_{m \alpha}}{\partial t_{n \beta}}=\int \frac{d \theta_{1} d \theta_{2}}{(2 \pi)^{2}} \underset{k}{\operatorname{res}}\left(\left(\mathcal{L}^{n} e^{i \beta \mathcal{U}}\right)_{\leq-1} \frac{\partial}{\partial k}\left(\mathcal{L}^{m} e^{i \alpha \mathcal{U}}\right)_{\geq 0}\right. \\
\left.-\left(\mathcal{L}^{m} e^{i \alpha \mathcal{U}}\right)_{\leq-1} \frac{\partial}{\partial k}\left(\mathcal{L}^{n} e^{i \beta \mathcal{U}}\right)_{\geq 0}\right) \\
=\int \frac{d \theta_{1} d \theta_{2}}{(2 \pi)^{2}} \underset{k}{\operatorname{res}}\left(\left(\mathcal{L}^{n} e^{i \beta \mathcal{U}}\right)_{\leq-1} \frac{\partial}{\partial k}\left(\mathcal{L}^{m} e^{i \alpha \mathcal{U}}\right)_{\geq 0}\right. \\
\left.+\left(\mathcal{L}^{n} e^{i \beta \mathcal{U}}\right)_{\geq 0} \frac{\partial}{\partial k}\left(\mathcal{L}^{n} e^{i \beta \mathcal{U}}\right)_{\leq-1}\right) .
\end{gathered}
$$

In the last line, the following lemma has been used.

\section{Lemma 3}

$$
\underset{k}{\operatorname{res}} f \frac{\partial g}{\partial k}=-\underset{k}{\operatorname{res}} g \frac{\partial f}{\partial k} .
$$

Proof Written in the language of contour integrals, this is nothing buth the formula of integration by parts.

Adding the obvious identities

$$
\begin{aligned}
\underset{k}{\operatorname{res}}\left(\mathcal{L}^{n} e^{i \beta \mathcal{U}}\right)_{\leq-1} \frac{\partial}{\partial k}\left(\mathcal{L}^{m} e^{i \alpha \mathcal{U}}\right)_{\leq-1} & =0 \\
\operatorname{res}_{k}\left(\mathcal{L}^{n} e^{i \beta \mathcal{U}}\right)_{\geq 0} \frac{\partial}{\partial k}\left(\mathcal{L}^{m} e^{i \alpha \mathcal{U}}\right)_{\geq 0} & =0
\end{aligned}
$$


to the above result, we can rewrite it into a more compact form,

$$
\frac{\partial h_{n \beta}}{\partial t_{m \alpha}}-\frac{\partial h_{m \alpha}}{\partial t_{n \beta}}=\int \frac{d \theta_{1} d \theta_{2}}{(2 \pi)^{2}} \underset{k}{\operatorname{res}}\left(\mathcal{L}^{n} e^{i \beta \mathcal{U}}\right) \frac{\partial}{\partial k}\left(\mathcal{L}^{m} e^{i \alpha \mathcal{U}}\right),
$$

and further using Lemma 2, but now in the inverse way, we can recast this into a $\lambda$-residue:

$$
=\int \frac{d \theta_{1} d \theta_{2}}{(2 \pi)^{2}} \operatorname{res}_{\lambda} a(\lambda, \mathcal{U}(\lambda), \mathcal{V}(\lambda)) \frac{\partial}{\partial \lambda} b(\lambda, \mathcal{U}(\lambda), \mathcal{V}(\lambda))
$$

3. We now have to evaluate the last integral. Since a similar issue also emerges in the proof of Theorem 6, let us formulate it in a somewhat general form as follows:

Lemma 4 Under the same assumption as in Theorem 4 , and for any LaurentFourier series $a(\lambda, u, v)$ and $b(\lambda, u, v)$ independent of $\mu$,

$$
\begin{aligned}
& \int \frac{d \theta_{1} d \theta_{2}}{(2 \pi)^{2}} \operatorname{res}_{\lambda} a(\lambda, \mathcal{U}(\lambda), \mathcal{V}(\lambda)) \frac{\partial}{\partial \lambda} b(\lambda, \mathcal{U}(\lambda), \mathcal{V}(\lambda)) \\
= & \int \frac{d u d v}{(2 \pi)^{2}} \operatorname{res}_{\lambda} a(\lambda, u, v) \frac{\partial}{\partial \lambda} b(\lambda, u, v) \\
& -\int \frac{d \theta_{1} d \theta_{2}}{(2 \pi)^{2}} \operatorname{res}_{\lambda} \mathcal{M}(\lambda)\{a(\lambda, u, v), b(\lambda, u, v)\}_{\theta} .
\end{aligned}
$$

Proof By the Leibniz rule, the integrand on the left hand side of (5.21) can be expanded into several pieces:

$$
\begin{aligned}
& a(\lambda, \mathcal{U}(\lambda), \mathcal{V}(\lambda)) \frac{\partial}{\partial \lambda} b(\lambda, \mathcal{U}(\lambda), \mathcal{V}(\lambda)) \\
= & \left.a(\lambda, \mathcal{U}(\lambda), \mathcal{V}(\lambda)) \frac{\partial b(\lambda, u, v)}{\partial \lambda}\right|_{u=\mathcal{U}(\lambda), v=\mathcal{V}(\lambda)} \\
& +a(\lambda, \mathcal{U}(\lambda), \mathcal{V}(\lambda))\left(\frac{\partial b(\lambda, u, v)}{\partial \mathcal{U}(\lambda)} \frac{\partial \mathcal{U}(\lambda)}{\partial \lambda}+\frac{\partial b(\lambda, u, v)}{\partial \mathcal{V}(\lambda)} \frac{\partial \mathcal{V}(\lambda)}{\partial \lambda}\right) .
\end{aligned}
$$

Contribution from the first part in the last two lines is given by

$$
\begin{aligned}
& \left.\int \frac{d \theta_{1} d \theta_{2}}{(2 \pi)^{2}} \operatorname{res}_{\lambda} a(\lambda, u, v) \frac{\partial b(\lambda, u, v)}{\partial \lambda}\right|_{u=\mathcal{U}(\lambda), v=\mathcal{V}(\lambda)} \\
= & \int \frac{d u d v}{(2 \pi)^{2}} \operatorname{res}_{\lambda} a(\lambda, u, v) \frac{\partial b(\lambda, u, v)}{\partial \lambda},
\end{aligned}
$$


where, by the topological assumption, we have changed the integration variables as $\left(\theta_{1}, \theta_{2}\right) \rightarrow(u, v)=(\mathcal{U}(\lambda), \mathcal{V}(\lambda))$. This gives the first term on the right hand side of (5.21). To evaluate the contribution from the second part, we note the identity

$$
\begin{aligned}
& \{\mathcal{M}(\lambda), b(\lambda, \mathcal{U}(\lambda), \mathcal{V}(\lambda))\}_{\theta} \\
= & \frac{\partial b(\lambda, u, v)}{\partial \mathcal{U}(\lambda)} \frac{\partial \mathcal{U}(\lambda)}{\partial \lambda}+\frac{\partial b(\lambda, u, v)}{\partial \mathcal{V}(\lambda)} \frac{\partial \mathcal{V}(\lambda)}{\partial \lambda}
\end{aligned}
$$

which one can easily show by (3.43). Contribution from the second part of (5.22) is thus given by the integral

$$
\int \frac{d \theta_{1} d \theta_{2}}{(2 \pi)^{2}} \operatorname{res}_{\lambda} a(\lambda, \mathcal{U}(\lambda), \mathcal{V}(\lambda))\{\mathcal{M}(\lambda), b(\lambda, \mathcal{U}(\lambda), \mathcal{V}(\lambda))\}_{\theta}
$$

which can be rewritten

$$
\begin{aligned}
= & \int \frac{d \theta_{1} d \theta_{2}}{(2 \pi)^{2}} \operatorname{res}_{\lambda}\{\mathcal{M}(\lambda) a(\lambda, \mathcal{U}(\lambda), \mathcal{V}(\lambda)), b(\lambda, \mathcal{U}(\lambda), \mathcal{V}(\lambda))\}_{\theta} \\
& -\int \frac{d \theta_{1} d \theta_{2}}{(2 \pi)^{2}} \operatorname{res}_{\lambda} \mathcal{M}(\lambda)\{a(\lambda, \mathcal{U}(\lambda), \mathcal{V}(\lambda)), b(\lambda, \mathcal{U}(\lambda), \mathcal{V}(\lambda))\}_{\theta}
\end{aligned}
$$

The first term first term vanishes by Lemma 1, and the second term gives the second term on the right hand side of (5.21). Thus (5.21) is proven.

Returning to the case under consideration, $a$ and $b$ are given by

$$
a(\lambda, u, v)=\lambda^{m} e^{i \alpha u}, \quad b(\lambda, u, v)=\lambda^{n} e^{i \beta u}
$$

Applying the above lemma to this case, we can readily see that the right hand side of (5.20) vanishes, so that (5.8) are indeed satisfied.

\subsection{Proof of the second theorem}

To prove Theorem 5, we have to show that

$$
\delta_{\mathcal{A}} \frac{\partial F}{\partial t_{m \alpha}}=\frac{\partial}{\partial t_{m \alpha}} \delta_{\mathcal{A}} F
$$


We calculate both hand sides separately, and show that they coincide. The method of calculations are almost parallel to the last subsection, simply $\partial / \partial t_{n \beta}$ being replaced by $\delta_{\mathcal{A}}$.

1. Let us consider the left hand side of (5.27). This is similar to the calculation of $\partial h_{m \alpha} / \partial t_{n \beta}$ in the last subsection. First, since $\delta_{\mathcal{A}}$ (like $\left.\partial / \partial t_{n \beta}\right)$ is also a derivation,

$$
\begin{aligned}
\delta_{\mathcal{A}} \frac{\partial F}{\partial t_{m \alpha}} & =\delta_{\mathcal{A}} \int \frac{d \theta_{1} d \theta_{2}}{(2 \pi)^{2}} \underset{\lambda}{\operatorname{res}} \lambda^{m} e^{i \alpha \mathcal{U}(\lambda)} \mathcal{M}(\lambda) \\
& =\int \frac{d \theta_{1} d \theta_{2}}{(2 \pi)^{2}} \underset{\lambda}{\operatorname{res}} \lambda^{m}\left(\delta_{\mathcal{A}} e^{i \alpha \mathcal{U}(\lambda)} \cdot \mathcal{M}(\lambda)+e^{i \alpha \mathcal{U}(\lambda)} \cdot \delta_{\mathcal{A}} \mathcal{M}(\lambda)\right),
\end{aligned}
$$

and evaluating the terms containing $\delta_{\mathcal{A}}$ by using the defining equation (4.24) and the Leibniz rule of $\delta_{\mathcal{A}}$, we find that

$$
\begin{gathered}
=\int \frac{d \theta_{1} d \theta_{2}}{(2 \pi)^{2}} \underset{\lambda}{\operatorname{res}} \lambda^{m}\left\{\mathcal{A}_{\leq-1}(\lambda), e^{i \alpha \mathcal{U}(\lambda)}\right\}_{\theta} \\
+\int \frac{d \theta_{1} d \theta_{2}}{(2 \pi)^{2}} \operatorname{res}_{\lambda} \lambda^{m} e^{i \alpha \mathcal{U}(\lambda)} \frac{\partial \mathcal{A}_{\leq-1}(\lambda)}{\partial \lambda} .
\end{gathered}
$$

The first term in the last two lines vanish because of Lemma 11. The second term can be rewritten into a $k$-residue by means of Lemma 2. Thus,

$$
\begin{aligned}
\delta_{\mathcal{A}} \frac{\partial F}{\partial t_{m \alpha}} & =\int \frac{d \theta_{1} d \theta_{2}}{(2 \pi)^{2}} \underset{k}{\operatorname{res}} \mathcal{L}^{m} e^{i \alpha \mathcal{U}} \frac{\partial}{\partial k} \mathcal{A}_{\leq-1} \\
& =\int \frac{d \theta_{1} d \theta_{2}}{(2 \pi)^{2}} \underset{k}{\operatorname{res}} \mathcal{B}_{m \alpha} \frac{\partial}{\partial k} \mathcal{A}_{\leq-1} \\
& =-\int \frac{d \theta_{1} d \theta_{2}}{(2 \pi)^{2}} \underset{k}{\operatorname{res}} \mathcal{A}_{\leq-1} \frac{\partial}{\partial k} \mathcal{B}_{m \alpha} .
\end{aligned}
$$

(Lemma 3) has been used in deriving the last line.)

2. Let us now consider the right hand side of (5.27). Differentiating the defining equation (5.3) of $\delta_{\mathcal{A}} F$, we first obtain

$$
\begin{aligned}
\frac{\partial}{\partial t_{m \alpha}} \delta_{\mathcal{A}} F= & -\frac{\partial}{\partial t_{m \alpha}} \int \frac{d \theta_{1} d \theta_{2}}{(2 \pi)^{2}} \operatorname{res}_{\lambda} \int_{0}^{\mathcal{M}(\lambda)} d \mu \mathcal{A}(\lambda, \mu, \mathcal{U}(\lambda), \mathcal{V}(\lambda)) \\
= & -\int \frac{d \theta_{1} d \theta_{2}}{(2 \pi)^{2}} \operatorname{res}_{\lambda} \mathcal{A}(\lambda, \mathcal{M}(\lambda), \mathcal{U}(\lambda), \mathcal{V}(\lambda)) \frac{\partial \mathcal{M}(\lambda)}{\partial t_{m \lambda}} \\
& -\int \frac{d \theta_{1} d \theta_{2}}{(2 \pi)^{2}} \operatorname{res}_{\lambda} \int_{0}^{\mathcal{M}(\lambda)} d \mu \frac{\partial}{\partial t_{m \alpha}} \mathcal{A}(\lambda, \mu, \mathcal{U}(\lambda), \mathcal{V}(\lambda)),
\end{aligned}
$$


and using (3.42) along with the chain rule of differentiation,

$$
\begin{aligned}
= & -\int \frac{d \theta_{1} d \theta_{2}}{(2 \pi)^{2}} \operatorname{res}_{\lambda} \mathcal{A}(\lambda, \mathcal{M}(\lambda), \mathcal{U}(\lambda), \mathcal{V}(\lambda)) \frac{\partial}{\partial \lambda} \mathcal{B}_{m \alpha}(\lambda) \\
& -\int \frac{d \theta_{1} d \theta_{2}}{(2 \pi)^{2}} \operatorname{res}_{\lambda} \mathcal{A}(\lambda, \mathcal{M}(\lambda), \mathcal{U}(\lambda), \mathcal{V}(\lambda))\left\{\mathcal{B}_{m \alpha}(\lambda), \mathcal{M}(\lambda)\right\}_{\theta} \\
& -\int \frac{d \theta_{1} d \theta_{2}}{(2 \pi)^{2}} \operatorname{res}_{\lambda} \int_{0}^{\mathcal{M}(\lambda)} d \mu \frac{\partial \mathcal{A}}{\partial \mathcal{U}(\lambda)}\left\{\mathcal{B}_{m \alpha}(\lambda), \mathcal{U}(\lambda)\right\}_{\theta} \\
& -\int \frac{d \theta_{1} d \theta_{2}}{(2 \pi)^{2}} \operatorname{res}_{\lambda} \int_{0}^{\mathcal{M}(\lambda)} d \mu \frac{\partial \mathcal{A}}{\partial \mathcal{V}(\lambda)}\left\{\mathcal{B}_{m \alpha}(\lambda), \mathcal{V}(\lambda)\right\}_{\theta} .
\end{aligned}
$$

Then by the same reasoning as used in the last subsection, the first term in the last four lines can be converted into a $k$-residue of the form

$$
-\int \frac{d \theta_{1} d \theta_{2}}{(2 \pi)^{2}} \underset{k}{\operatorname{res}} \mathcal{A} \frac{\partial}{\partial k} \mathcal{B}_{m \alpha}=-\int \frac{d \theta_{1} d \theta_{2}}{(2 \pi)^{2}} \underset{k}{\operatorname{ras}} \mathcal{A}_{\leq-1} \frac{\partial}{\partial k} \mathcal{B}_{m \alpha}
$$

The remaining part can be written as an integral of a single Poisson bracket of the form (which vanishes by Lemma 1):

$$
-\int \frac{d \theta_{1} d \theta_{2}}{(2 \pi)^{2}} \operatorname{res}_{\lambda}\left\{\mathcal{B}_{m \alpha}(\lambda), \int_{0}^{\mathcal{M}(\lambda)} d \mu \mathcal{A}(\lambda, \mu, \mathcal{U}(\lambda), \mathcal{V}(\lambda))\right\}=0
$$

Therefore,

$$
\frac{\partial}{\partial t_{m \alpha}} \delta_{\mathcal{A}} F=-\int \frac{d \theta_{1} d \theta_{2}}{(2 \pi)^{2}} \underset{k}{\operatorname{res}} \mathcal{A}_{\leq-1} \frac{\partial}{\partial k} \mathcal{B}_{m \alpha}
$$

Thus (5.29) and (5.33) turn out to give an identical result, completing the proof of (5.27).

\subsection{Proof of the third theorem}

We now prove Theorem [6. It is sufficient to prove the special relation

$$
\left[\delta_{\mathcal{A}}, \delta_{\mathcal{B}}\right] F=\delta_{\{\mathcal{A}, \mathcal{B}\}_{\lambda \mu u v}} F+c(\mathcal{A}, \mathcal{B}) \text {. }
$$

The following proof resembles the proof of Theorem $甘$ (and, indeed, includes it as a special case!). 
1. We first calculate $\delta_{\mathcal{A}} \delta_{\mathcal{B}} F$. Details are similar to the previous calculations. First, since $\delta_{\mathcal{A}}$ (like $\partial / \partial t_{m \alpha}$ ) is also a derivation, its action on the integral representation of $\delta_{\mathcal{B}} F$ can be evaluated as:

$$
\begin{aligned}
\delta_{\mathcal{A}} \delta_{\mathcal{B}} F & =-\delta_{\mathcal{A}} \int \frac{d \theta_{1} d \theta_{2}}{(2 \pi)^{2}} \underset{\lambda}{\operatorname{res}} \int_{0}^{\mathcal{M}(\lambda)} d \mu \mathcal{B}(\lambda, \mu, \mathcal{U}(\lambda), \mathcal{V}(\lambda)) \\
& =-\int \frac{d \theta_{1} d \theta_{2}}{(2 \pi)^{2}} \operatorname{res}_{\lambda} \mathcal{B}(\lambda, \mathcal{M}(\lambda), \mathcal{U}(\lambda), \mathcal{V}(\lambda)) \delta_{\mathcal{A}} \mathcal{M}(\lambda) \\
& =-\int \frac{d \theta_{1} d \theta_{2}}{(2 \pi)^{2}} \operatorname{res}_{\lambda} \int_{0}^{\mathcal{M}(\lambda)} d \mu \delta_{\mathcal{A}} \mathcal{B}(\lambda, \mu, \mathcal{U}(\lambda), \mathcal{V}(\lambda)) .
\end{aligned}
$$

Second, using the defining equation (5.3) and the Leibniz rule of $\delta_{\mathcal{A}}$ again, we can rewrite the above formula into the following form:

$$
\begin{aligned}
\delta_{\mathcal{A}} \delta_{\mathcal{B}} F= & -\int \frac{d \theta_{1} d \theta_{2}}{(2 \pi)^{2}} \operatorname{res}_{\lambda}\left\{\mathcal{A}_{\leq-1}(\lambda), \int_{0}^{\mathcal{M}(\lambda)} d \mu \mathcal{B}(\lambda, \mu, \mathcal{U}(\lambda), \mathcal{V}(\lambda))\right\}_{\theta} \\
& -\int \frac{d \theta_{1} d \theta_{2}}{(2 \pi)^{2}} \operatorname{res}_{\lambda} \mathcal{B}(\lambda, \mathcal{M}(\lambda), \mathcal{U}(\lambda), \mathcal{V}(\lambda)) \frac{\partial \mathcal{A}_{\leq-1}(\lambda)}{\partial \lambda}
\end{aligned}
$$

Now by the same reasoning as in the previous proofs, the first term vanishes and the second term can be rewritten into a $k$-residue. Thus we obtain the following expression of $\delta_{\mathcal{A}} \delta_{\mathcal{B}} F$ :

$$
\delta_{\mathcal{A}} \delta_{\mathcal{B}} F=-\int \frac{d \theta_{1} d \theta_{2}}{(2 \pi)^{2}} \underset{k}{\operatorname{res}} \mathcal{B}(\mathcal{L}, \mathcal{M}, \mathcal{U}, \mathcal{V})_{\geq 0} \frac{\partial}{\partial k} \mathcal{A}(\mathcal{L}, \mathcal{M}, \mathcal{U}, \mathcal{V})_{\leq-1}
$$

2. Now Subtract from the last equation the same equation with $\mathcal{A}$ and $\mathcal{B}$ interchanged. Also use Lemma 3 to reverse the order of the two terms sandwitching $\partial / \partial k$. By basically the same calculations as in the preceding sections, we eventually obtain

$$
\left[\delta_{\mathcal{A}}, \delta_{\mathcal{B}}\right] F=\int \frac{d \theta_{1} d \theta_{2}}{(2 \pi)^{2}} \operatorname{res}_{k} \mathcal{A}(\mathcal{L}, \mathcal{M}, \mathcal{U}, \mathcal{V}) \frac{\partial}{\partial k} \mathcal{B}(\mathcal{L}, \mathcal{M}, \mathcal{U}, \mathcal{V})
$$

and further by Lemma 2 , this can be converted into a $\lambda$-residue, i.e.,

$$
=\int \frac{d \theta_{1} d \theta_{2}}{(2 \pi)^{2}} \operatorname{res}_{\lambda} \mathcal{A}(\lambda, \mathcal{M}(\lambda), \mathcal{U}(\lambda), \mathcal{V}(\lambda)) \frac{\partial}{\partial \lambda} \mathcal{B}(\lambda, \mathcal{M}(\lambda), \mathcal{U}(\lambda), \mathcal{V}(\lambda)) \text {. }
$$


3. We now have to evaluate the last line of (5.38). Since target equation (5.34) is bilinear in $\mathcal{A}$ and $\mathcal{B}$, it is sufficient to consider the case where $\mathcal{A}$ and $\mathcal{B}$ take the following somewhat special form:

$$
\mathcal{A}=a(\lambda, u, v) \mu^{j}, \quad \mathcal{B}=b(\lambda, u, v) \mu^{k} \quad(j, k \geq 0) .
$$

(i) The case where $j+k=0$ (i.e., $j=k=0$ ): In this case, the integrand of the last line of (5.38) takes exactly the same for as in Lemma 0 . The first term on the right hand side of (5.21) now gives the cocycle $c(\mathcal{A}, \mathcal{B})$ :

$$
\int \frac{d u d v}{(2 \pi)^{2}} \underset{\lambda}{\operatorname{ras}} a(\lambda, u, v) \frac{\partial}{\partial \lambda} b(\lambda, u, v)=c(a, b) .
$$

The last term of (5.21), meanwhile, gives $\delta_{\{a, b\}_{\lambda \mu u v}} F$ :

$$
\begin{aligned}
& -\int \frac{d \theta_{1} d \theta_{2}}{(2 \pi)^{2}} \underset{\lambda}{\operatorname{ras}} \mathcal{M}(\lambda)\{a(\lambda, \mathcal{U}(\lambda), \mathcal{V}(\lambda)), b(\lambda, \mathcal{U}(\lambda), \mathcal{V}(\lambda))\}_{\theta} \\
= & -\int \frac{d \theta_{1} d \theta_{2}}{(2 \pi)^{2}} \underset{\lambda}{\operatorname{res}} \int_{0}^{\mathcal{M}(\lambda)} d \mu\{a(\lambda, \mathcal{U}(\lambda), \mathcal{V}(\lambda)), b(\lambda, \mathcal{U}(\lambda), \mathcal{V}(\lambda))\}_{\theta} \\
= & \delta_{\{a, b\}_{\lambda \mu u v}} F .
\end{aligned}
$$

(In the last line, we we have used the identity

$$
\{a(\lambda, \mathcal{U}(\lambda), \mathcal{V}(\lambda)), b(\lambda, \mathcal{U}(\lambda), \mathcal{V}(\lambda))\}_{\theta}=\left.\{a, b\}_{\lambda \mu u v}\right|_{u=\mathcal{U}(\lambda), v=\mathcal{V}(\lambda)},
$$

which follows from the first equation of (3.43).) Collecting these pieces, one can see that (5.34) is indeed satisfied in this case.

(ii) The case where $j+k>0$ : Now the integrand of (5.38) becomes

$$
\begin{aligned}
& \mathcal{A}(\lambda, \mathcal{M}(\lambda), \mathcal{U}(\lambda), \mathcal{V}(\lambda)) \frac{\partial}{\partial \lambda} \mathcal{B}(\lambda, \mathcal{M}(\lambda), \mathcal{U}(\lambda), \mathcal{V}(\lambda)) \\
= & k \mathcal{M}(\lambda)^{j+k-1} \frac{\partial \mathcal{M}(\lambda)}{\partial \lambda} a(\lambda, \mathcal{U}(\lambda), \mathcal{V}(\lambda)) b(\lambda, \mathcal{U}(\lambda), \mathcal{V}(\lambda)) \\
& +\mathcal{M}(\lambda)^{j+k} a(\lambda, \mathcal{U}(\lambda), \mathcal{V}(\lambda)) \frac{\partial}{\partial \lambda} b(\lambda, \mathcal{U}(\lambda), \mathcal{V}(\lambda)) \\
= & \frac{\partial}{\partial \lambda}\left(\frac{k}{j+k} \mathcal{M}(\lambda)^{j+k} a(\mathcal{U}(\lambda), \mathcal{V}(\lambda)) b(\mathcal{U}(\lambda), \mathcal{V}(\lambda))\right) \\
& +\frac{j}{j+k} \mathcal{M}(\lambda)^{j+k} a(\lambda, \mathcal{U}(\lambda), \mathcal{V}(\lambda)) \frac{\partial}{\partial \lambda} b(\lambda, \mathcal{U}(\lambda), \mathcal{V}(\lambda)) \\
& -\frac{k}{j+k} \mathcal{M}(\lambda)^{j+k} b(\lambda, \mathcal{U}(\lambda), \mathcal{V}(\lambda)) \frac{\partial}{\partial \lambda} a(\lambda, \mathcal{U}(\lambda), \mathcal{V}(\lambda)) .
\end{aligned}
$$


The first term in the last three lines vanishes under the $\lambda$-residue (because this is a total derivative with respect to $\lambda$ ). The $\lambda$-derivatives in the other terms can be evaluated by the chain rule and the second and third equations of (3.43):

$$
\begin{aligned}
\frac{\partial}{\partial \lambda} a(\lambda, \mathcal{U}(\lambda), \mathcal{V}(\lambda))= & \left.\frac{\partial a(\lambda, u, v)}{\partial \lambda}\right|_{u=\mathcal{U}(\lambda), v=\mathcal{V}(\lambda)} \\
& +\{\mathcal{M}(\lambda), a(\lambda, \mathcal{U}(\lambda), \mathcal{V}(\lambda))\}_{\theta}, \\
\frac{\partial}{\partial \lambda} b(\lambda, \mathcal{U}(\lambda), \mathcal{V}(\lambda))= & \left.\frac{\partial b(\lambda, u, v)}{\partial \lambda}\right|_{u=\mathcal{U}(\lambda), v=\mathcal{V}(\lambda)} \\
& +\{\mathcal{M}(\lambda), b(\lambda, \mathcal{U}(\lambda), \mathcal{V}(\lambda))\}_{\theta}
\end{aligned}
$$

Plugging these formulas into the right hand side of (5.43), we obtain four terms originating in the four terms on the right hand side of (5.44). We now separate them into two groups: (a) those coming from the first terms on the right hand side of (5.44), and (b) those from the second terms thereof.

(a) Contribution of these terms to (5.43) can be written

$$
\begin{aligned}
& \left(\frac{j}{j+k} \mathcal{M}(\lambda)^{j+k} a(\lambda, u, v) \frac{\partial b(\lambda, u, v)}{\partial \lambda}\right. \\
& \left.-\frac{k}{j+k} \mathcal{M}(\lambda)^{j+k} b(\lambda, u, v) \frac{\partial a(\lambda, u, v)}{\partial \lambda}\right)\left.\right|_{u=\mathcal{U}(\lambda), v=\mathcal{V}(\lambda)} \\
= & -\left.\int_{0}^{\mathcal{M}(\lambda)} d \mu\{\mathcal{A}(\lambda, \mu, u, v), \mathcal{B}(\lambda, \mu, u, v)\}_{\lambda \mu}\right|_{u=\mathcal{U}(\lambda), v=\mathcal{V}(\lambda)} .
\end{aligned}
$$

(b) Contribution of these terms to (5.43) is given by

$$
\begin{aligned}
& \frac{j}{j+k} \mathcal{M}(\lambda)^{j+k} a(\lambda, \mathcal{U}(\lambda), \mathcal{V}(\lambda))\{\mathcal{M}(\lambda), b(\lambda, \mathcal{U}(\lambda), \mathcal{V}(\lambda))\}_{\theta} \\
& -\frac{k}{j+k} \mathcal{M}(\lambda)^{j+k} b(\lambda, \mathcal{U}(\lambda), \mathcal{V}(\lambda))\{\mathcal{M}(\lambda), a(\lambda, \mathcal{U}(\lambda), \mathcal{V}(\lambda))\}_{\theta} \\
= & \frac{j}{j+k} a(\lambda, \mathcal{U}(\lambda), \mathcal{V}(\lambda))\left\{\frac{\mathcal{M}(\lambda)^{j+k+1}}{j+k+1}, b(\lambda, \mathcal{U}(\lambda), \mathcal{V}(\lambda))\right\}_{\theta} \\
& -\frac{k}{j+k} b(\lambda, \mathcal{U}(\lambda), \mathcal{V}(\lambda))\left\{\frac{\mathcal{M}(\lambda)^{j+k+1}}{j+k+1}, a(\lambda, \mathcal{U}(\lambda), \mathcal{V}(\lambda))\right\}_{\theta}
\end{aligned}
$$




$$
\begin{aligned}
= & \left\{\frac{j}{(j+k)(j+k+1)} a(\lambda, \mathcal{U}(\lambda), \mathcal{V}(\lambda)) \mathcal{M}(\lambda)^{j+k+1}, b(\lambda, \mathcal{U}(\lambda), \mathcal{V}(\lambda))\right\}_{\theta} \\
& -\left\{\frac{k}{(j+k)(j+k+1)} b(\lambda, \mathcal{U}(\lambda), \mathcal{V}(\lambda)) \mathcal{M}(\lambda)^{j+k+1}, a(\lambda, \mathcal{U}(\lambda), \mathcal{V}(\lambda))\right\}_{\theta} \\
& -\{a(\lambda, \mathcal{U}(\lambda), \mathcal{V}(\lambda)), b(\lambda, \mathcal{U}(\lambda), \mathcal{V}(\lambda))\}_{\theta} \frac{\mathcal{M}(\lambda)^{j+k+1}}{j+k+1}
\end{aligned}
$$

The first two terms in the last three lines vanish in the $\theta$-integral (Lemma 1). The last term can be rewritten

$$
\begin{aligned}
& -\left.\{a(\lambda, u, v), b(\lambda, u, v)\}_{u v} \frac{\mu^{j+k+1}}{j+k+1}\right|_{\mu=\mathcal{M}(\lambda), u=\mathcal{U}(\lambda), v=\mathcal{V}(\lambda)} \\
= & -\left.\int_{0}^{\mathcal{M}(\lambda)} d \mu\{A(\lambda, \mu, u, v), B(\lambda, \mu, u, v)\}_{u v}\right|_{u=\mathcal{U}(\lambda), v=\mathcal{V}(\lambda)} .
\end{aligned}
$$

Thus, contribution from each term of (5.43) has been specified by (5.44), (5.45) and (5.47). Inserting these pieces, the integral of (5.38) can now be written

$$
\begin{aligned}
& \int \frac{d \theta_{1} d \theta_{2}}{(2 \pi)^{2}} \operatorname{res}_{\lambda} \mathcal{A}(\lambda, \mathcal{M}(\lambda), \mathcal{U}(\lambda), \mathcal{V}(\lambda)) \frac{\partial}{\partial \lambda} \mathcal{B}(\lambda, \mathcal{M}(\lambda), \mathcal{U}(\lambda), \mathcal{V}(\lambda)) \\
= & -\left.\int \frac{d \theta_{1} d \theta_{2}}{(2 \pi)^{2}} \operatorname{res}_{\lambda} \int_{0}^{\mathcal{M}(\lambda)} d \mu\{\mathcal{A}(\lambda, \mu, u, v), \mathcal{B}(\lambda, \mu, u, v)\}\right|_{u=\mathcal{U}(\lambda), v=\mathcal{V}(\lambda)} \\
= & \delta_{\{\mathcal{A}, \mathcal{B}\}_{\lambda \mu u v}} F .
\end{aligned}
$$

Meanwhile, since $\mathcal{A}(\lambda, 0, u, v)=\mathcal{B}(\lambda, 0, u, v)=0$, the cocycle itself vanishes: $c(\mathcal{A}, \mathcal{B})=0$. Thus (5.34) turns out to be satisfied in this case, too.

\section{Conclusion}

We have considered a new higher dimensional analogue of the dispersionless KP hierarchy — the toroidal model. The structure of this model is almost parallel to the planar model In our previous paper. These higher dimensional hierarchies have extra spatial dimensions in addition to the two-dimensional "phase space" variables $(k, x)$ of the dispersionless KP hierarchy. In the toroidal model, they are compactified to a two (or any even) dimensional 
torus. Because of this, the Poisson algebra has a "trace functional" as mentioned in Introduction. With this linear functional, we have been able to introduce an analogue of the $F$ function of the dispersionless KP hierarchy. We have also constructed an infinite number of additional symmetries, and shown that their commutation relations obey an underlying higher dimensional Poisson algebraic structure. The $F$ function induces an anomalous term in commutation relations of additional symmetries, which strongly suggests that our definition of the $F$ function is a natural generalization of the $F$ functions in lower dimensional dispersionless integrable hierarchies.

Let us conclude this paper by pointing out two issues that should be elucidated on the bases of our results.

1. Field theoretic meaning of $\delta_{\mathcal{A}} F$. In the case of the dispersionless KP hierarchy, we have a phase space integral representation, (2.27), of $\delta_{\mathcal{A}} F$. This expression is reminiscent of the "fermi fluid picture" [22] of $c=1$ matrix models. Actually, they both stem from an underlying $1+1$ dimensional free fermion system. In the case of (2.27), the curve $\mu=\mathcal{M}(\lambda)$ in the $(\lambda, \mu)$ space represent a "fermi surface", and the curve $\mu=0$ probably the bottom of the "fermi sea".

Remarkably, we can rewrite the defining equation (5.3) of $\delta_{\mathcal{A}}$ in the toroidal model, too, can be rewritten into a similar multiple integral:

$$
\delta_{\mathcal{A}} F=-\int \frac{d \theta_{1} d \theta_{2}}{(2 \pi)^{2}} \oint \frac{d \lambda}{2 \pi i} \int_{0}^{\mathcal{M}(\lambda)} d \mu \mathcal{A}(\lambda, \mu, \mathcal{U}(\lambda), \mathcal{V}(\lambda)) .
$$

This poses an interesting question: What is a higher dimensional counterpart of the $1+1$-dimensional free fermion theory? This should be a kind of large- $N$ limit of the $N$-component fermion system in the $N$-component $\mathrm{KP}$ hierarchy [3], but because of the "multiplicative renormalization" mentioned in Introduction, the fermion system seems to turn into a substantially distinct (stringy?) object.

2. Moyal algebraic algebraic version of toroidal model. The planar model has a Moyal algebraic version [1]. It is not hard to construct a Moyal algebraic version of the toroidal model. Since the toroidal Moyal algebra has a "trace functional" as mentioned in Introduction, we have a chance to construct a tau function of this hierarchy. If the tau function defined by any means, the $F$ function will be characterized as a leading term of $\hbar$-expansion

$$
\tau(\hbar, t, x)=\exp \left(-\hbar^{-2} F(t, x)+O\left(\hbar^{-1}\right)\right) .
$$


This issue is also related to the previous one, i.e., searching for a field theoretic representation of higher dimensional integrable hierarchies. It is well known that the tau functions of the ordinary and multi-component KP hierarchies can be written as an infinite determinant [2, 1 . The hypothetical tau (or $F$ ) function of our higher dimensional hierarchy, too, might be a determinant on a huge vector space. Actually, since the trace functional on a Moyal (or Poisson) algebra is a multiplicatively renormalized large- $N$ limit of the ordinary trace on $\mathrm{gl}(N)$, this "determiant" (unlike the relatively naive infinite determinant for the KP hierarchy) should be a considerably complicated quantity (a "renormalized determinant", so to speak).

\section{References}

[1] Takasaki, K., Nonabelian KP hierarchy with Moyal algebraic coefficients, KUCP-0062, hep-th/9305169 (May, 1993), J. Geom. Phys. 14 (to appear).

[2] Sato, M., and Sato, Y., Soliton equations as dynamical systems in an infinite dimensional Grassmann manifold, in: Nonlinear Partial Differential Equations in Applied Sciences (North-Holland, Amsterdam, 1982).

[3] Date, E., Jimbo, M., Kashiwara, M., and Miwa, T., Transformation groups for soliton equations, III, J. Phys. Soc. Japan 50 (1981), 38063612; Transformation groups for soliton equations, in: Nonlinear Integrable Systems - Classical Theory and Quantum Theory (World Scientific, Singapore, 1983).

[4] Segal, G., and Wilson, G., Loop groups and equations of KdV type, Inst. Hautes Etudes Sci. Publ. Math. 63 (1985), 1-64.

[5] Plebanski, J.F., Some solutions of complex Einstein equations, J. Math. Phys. 16 (1975), 2395-2402.

[6] Krichever, I.M., The dispersionless Lax equations and topological minimal models, Commun. Math. Phys. 143 (1991), 415-426; The $\tau$-function of the universal Whitham hierarchy, matrix models and topological field theories, Comm. Pure. Appl. Math. 47 (1994), 437-475. 
[7] Dubrovin, B.A., Integrable systems in topological field theory, Nucl. Phys. B379 (1992), 627-689; Hamiltonian formalism of Whitham-type hierarchies and topological Landau-Ginsburg models, Commun. Math. Phys. 145 (1992), 195-207.

[8] Takasaki, K., and Takebe, T., SDiff(2) KP hierarchy, Int. J. Mod. Phys. A7, Suppl. 1B (1992), 889-922.

[9] Boyer, C.P., and Plebanski, J.F., An infinite hierarchy of conservation laws and nonlinear superposition principles for self-dual Einstein spaces, J. Math. Phys. 26 (1985), 229-234.

[10] Takasaki, K., Symmetries of hyper-Kähler (or Poisson gauge field) hierarchy, J. Math. Phys. 31 (1990), 1877-1888.

[11] Park, Q-H., Extended conformal symmetries in real heavens, Phys. Lett. 236B (1990), 429-432; Self-dual gravity as a large- $N$ limit of the $2 \mathrm{D}$ nonlinear sigma model, Phys. Lett. 238B (1990), 287-290.

[12] Fairlie, D., Fletcher, P., and Zachos, C.K., Trigonometric structure constants for new infinite algebras, Phys.Lett.B218 (1989), 203.

Fairlie, D.B., Fletcher, P., and Zachos, C.K., Infinite-dimensional algebras and trigonometric basis for the classical Lie algebras, J. Math. Phys. 31 (1990), 1088-1094.

Hoppe, J., Olshanetsky, M., and Theisen, S., Dynamical systems on quantum tori algebras, Commun. Math. Phys. 155 (1993), 429-448.

[13] Hoppe, J., Quantum theory of a massless relativistic surface, Ph.D. thesis (MIT, 1982); Elem. Part. Res. J. (Kyoto) 80 (1989), 145-202.

Fairlie, D.B., and Zachos, C.K., Infinite-dimensional algebras, sine brackets, and SU( $\infty)$, Phys. Lett. 224B (1989), 101-107.

Pope, C.N., and Stelle, K.S., SU( $\infty)$, $\mathrm{SU}_{+}(\infty)$ and area-preserving algebras, Phys. Lett. 226B (1989), 257-263.

[14] Strachan, I.A.B., The Moyal algebra and integrable deformations of the self-dual Einstein equations, Phys. Lett. B282 (1992), 63-66.

Takasaki, K., Dressing operator approach to Moyal algebraic deformation of selfdual gravity, KUCP-0054, hep-th/9212103 (December, 1994), J. Geom. Phys. 14 (to appear). 
Castro, C., A universal $W_{\infty}$ algebra and quantization of integrable deformations of self dual gravity, IAEC-2-94 (February, 1994); Nonlinear $W_{\infty}$ algebras from nonlinear integrable deformations of self dual gravity, IAEC-4-94 (May, 1994).

[15] Jimbo, M., Miwa, T., and Ueno, K., Monodromy preserving deformations of linear ordinary differential equations with rational coefficients I, Physica 2D (1981), 306-352.

Jimbo, M., and Miwa, T., ditto II, Physica 2D (1981), 407-448; ditto II, Physica 4D (1981), 26-46.

[16] Takasaki, K., and Takebe, T., Quasi-classical limit of KP hierarchy, Wsymmetries and free fermions, KUCP-0050/92, hep-th/9207081 (July, 1992), Proceedings of Lobachevsky Semester 1992 St. Petersburg (to appear).

[17] Takasaki, K., and Takebe, T., Integrable hierarchies and dispersionless limit, UTMS 94-35, hep-th/9405096 (May 1995).

[18] Orlov, A.Yu., and Shulman, E.I., Additional symmetries for integrable equations and conformal algebra representation, Lett. Math. Phys. 12 (1986), 171-179.

[19] Orlov, A.Yu., Vertex operators, $\bar{\partial}$-problems, symmetries, variational indentities and Hamiltonian formalism for $2+1$ integrable systems, in: Plasma Theory and Nonlinear and Turbulent Processes in Physics (World Scientific, Singapore, 1988).

Grinevich, P.G., and Orlov, A.Yu., Virasoro action on Riemann surfaces, Grassmannians, $\operatorname{det} \bar{\partial}_{j}$ and Segal Wilson $\tau$ function, in: Problems of Modern Quantum Field Theory (Springer-Verlag, 1989).

[20] Penrose, R., Nonlinear gravitons and curved twistor theory, Gen. Rel. Grav. 7 (1976), 31-52.

Newman, E.T., Porter, J.R., and Tod, K.P., Twistor surfaces and rightflat spaces, Gen. Rel. Grav. 9 (1978), 1129-1142.

Gindikin, S.G., Integral geometry and twistors, in: Twistor Geometry and Non-linear Systems, Lecture Notes in Mathematics vol. 970 (Springer-Verlag, 1982).

Hitchin, N.J., Complex manifolds and Einstein's equations, ibid. 
[21] Takasaki, K., and Takebe, T., SDiff(2) Toda equation - hierarchy, tau function and symmetries, Lett. Math. Phys. 23 (1991), 205-214.

[22] Polchinski, J., Classical limit of $(1+1)$-dimensional string theory, Nucl. Phys. B326 (1991), 125-140.

Dhar, A., Mandal, G., and Wadia, S.R., Classical Fermi fluid and geometric action for $c=1$, Int. J. Mod. Phys. A8 (1993), 325-350.

Iso, S., Karabali, D., and Sakita, B., One-dimensional Fermions and two-dimensional droplets via Chern-Simons theory, Nucl. Phys. B388 (1992), 700-714.

[23] Kodama, Y., and Gibbons, J., Integrability of the dispersionless KP hierarchy, in: Nonlinear and Turbulent Processes in Physics (World Scientific, 1990).

[24] Park, Q-Han, 2-D sigma model approach to 4-D instantons, Int. J. Mod. Phys. A7 (1992), 1415-1448. 